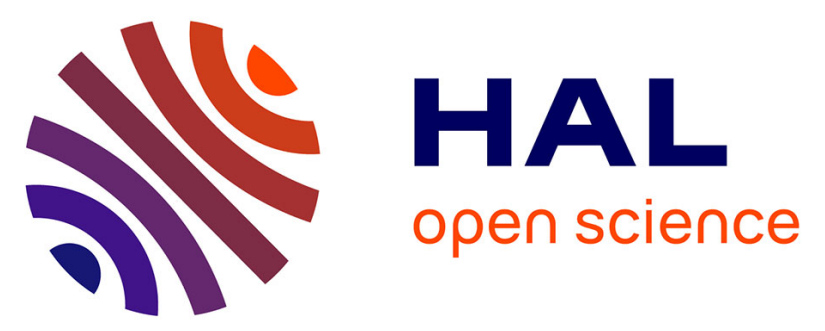

\title{
Exploring few-photon, few-electron reactions at FLASH: from ion yield and momentum measurements to time-resolved and kinematically complete experiments
}

\author{
A Rudenko, y H Jiang, M Kurka, K U Kühnel, L Foucar, O Herrwerth, M \\ Lezius, M F Kling, C D Schröter, R Moshammer, et al.
}

\section{To cite this version:}

A Rudenko, y H Jiang, M Kurka, K U Kühnel, L Foucar, et al.. Exploring few-photon, few-electron reactions at FLASH: from ion yield and momentum measurements to time-resolved and kinematically complete experiments. Journal of Physics B: Atomic, Molecular and Optical Physics, 2010, 43 (19), pp.194004. 10.1088/0953-4075/43/19/194004 . hal-00569836

\section{HAL Id: hal-00569836 https://hal.science/hal-00569836}

Submitted on 25 Feb 2011

HAL is a multi-disciplinary open access archive for the deposit and dissemination of scientific research documents, whether they are published or not. The documents may come from teaching and research institutions in France or abroad, or from public or private research centers.
L'archive ouverte pluridisciplinaire HAL, est destinée au dépôt et à la diffusion de documents scientifiques de niveau recherche, publiés ou non, émanant des établissements d'enseignement et de recherche français ou étrangers, des laboratoires publics ou privés. 


\title{
Exploring few-photon, few-electron reactions at FLASH: From ion yield and momentum measurements to time-resolved and kinematically complete experiments
}

\author{
A. Rudenko' ${ }^{1}$, Y.H. Jiang ${ }^{2}$, M. Kurka ${ }^{2}$, K.U. Kühnel ${ }^{2}$, L. Foucar ${ }^{1}$, O. Herrwerth ${ }^{3}$, M. Lezius ${ }^{3}$, \\ M.F. Kling ${ }^{3}$, C.D. Schröter ${ }^{2}$, R. Moshammer ${ }^{1,2}$ and J. Ullrich ${ }^{1,2}$ \\ ${ }^{1}$ Max-Planck Advanced Study Group at CFEL, 22607 Hamburg, Germany \\ ${ }^{2}$ Max-Planck-Institut für Kernphysik, 69117 Heidelberg, Germany \\ ${ }^{3}$ Max-Planck-Institut für Quantenoptik, 85748 Garching, Germany
}

32.80.Rm, 41.60.Cr, 42.65.-k

\begin{abstract}
:
In this contribution we briefly review a series of recent pioneering experiments on fewphoton-induced multiple fragmentation of atoms and molecules performed at the Free electron LASer at Hamburg (FLASH) using a "Reaction Microscope", i.e., a coincident momentum imaging technique. For atoms we consider the most basic non-linear process, namely direct or sequential two-photon double ionization (TPDI). In particular benchmark data for theory are presented such as recoil-ion momentum distributions for direct TPDI of $\mathrm{He}$ and Ne, and fully differential data for sequential TPDI of Ne. For molecules we show how one can identify contributions from different reaction channels by inspecting the measured kinetic energy and angular distributions of ionic fragments, and even infer the time-delay between the two photon absorption events in TPDI by detailed comparison with theory. Finally, we describe a novel split mirror arrangement for EUV-pump - EUV-probe experiments, and present the very first time-resolved data on the dynamics of $\mathrm{N}_{2}$ molecules irradiated by the FLASH light.
\end{abstract}




\section{Introduction}

Light-induced nonlinear reactions were envisioned as early as 1931, when M. GöppertMayer predicted that the energies of more than one photon can be combined to release a bound electron from an atom [1]. Although the first signatures of two-photon processes had been observed in 1950 in the radio frequency absorption spectra of molecular RbF [2], it took until the invention of the laser to provide experimental evidences of multiphoton ionization [3]. Nearly at the same time it was realized that mechanisms of nonlinear ionization crucially depends on both, the frequency as well as the intensity of light, and basic theoretical concepts for our current understanding of strong-field-induced phenomena were established [4-7]. In particular, in the pioneering work of Keldysh quantitative conditions for the applicability of the 'photon' or 'field' description of non-linear optical processes have been derived [4].

With the availability of laser sources at even higher intensities, it became possible to eject more than one electron from an atom exposed to an external light field. Since the first observation of the doubly charged ions produced by multiphoton absorption [8], manyelectron dynamics and electron-electron correlation in intense laser pulse interactions with matter have become one of the hottest topics in atomic, molecular and optical physics $[9,10]$, along with studies of few-electron ejection upon single photon absorption $[11,12]$. Whereas the latter process, where the electrons have to share the energy of a single light quantum, is possible exclusively due to electron-electron correlation, it turned out that in case of strong field double or multiple ionization both independent ("sequential") or correlated ("direct", or "non-sequential") electron emission occurs, depending on the parameters of the light field and atomic or molecular structure. In particular, field-mediated correlation ("recollision") $[13,14]$ was found to be of decisive importance at moderate field intensities, often enhancing multiple ionization cross sections for linearly polarized light by many orders of magnitude observed in yield measurements of multiple charged ions [15-18]. Still, the subtle details of 
the underlying dynamics are not completely settled and, thus, subject of continuing experimental as well as theoretical effort (see e.g. [19-22]).

Until very recently studies of nonlinear few-electron processes have been restricted to the optical and infrared domain, where the Keldysh parameter [4] $\gamma=\sqrt{I_{P} / U_{P}}$ is often close to or smaller than unity (here $\mathrm{I}_{\mathrm{p}}$ is the ionization potential and $U_{p}=I / 4 \omega^{2}$ is the ponderomotive potential, I the light intensity and $\omega$ it's frequency). In the first decade of the $21^{\text {st }}$ century the rapid development of laser- (high-harmonic generation, HHG) [23,24] and accelerator-based (free-electron lasers, FEL) [25-28] light sources enabled generating intense, ultrashort VUV, EUV, and even X-ray pulses. Those in turn allowed one to extend experiments on multiphoton - multi-electron reactions towards shorter wavelengths [29-48]. Due to their fundamental nature and also in a view of practical applications (since multi-photon processes occur whenever intense FEL radiation interacts with matter, i.e., with atoms, molecules, clusters or solids) enormous theoretical interest has emerged to describe basic few-photon few-electron reactions for simple atomic and molecular systems (see [49-69] for an incomplete selection of recent publications). Moreover, these novel light sources currently delivering pulses shorter than ten femtoseconds with realistic prospects of reaching the sub-fs regime (FELs), or even less than hundred attoseconds (HHG), are paving the way for a variety of novel time-resolved experiments. They will substantially extend the boarders of present ultrafast science, not only in terms of the time resolution that can be achieved stretching towards the attosecond domain ([70-72], see [24] for a recent review), and new electronic states that can be accessed with EUV or X-ray pulses $[70,73,74]$ but, moreover, in terms of techniques visualizing the dynamics. In particular, time-resolved X-ray diffraction [75] or electron holography [76] experiments nurture the hope of recording the "molecular movie", i.e. tracing in time the nuclear motion during a chemical reaction, and threedimensional imaging of photo-electrons emerging upon absorption of a EUV- or X-ray probe 
pulse will enable to directly follow the evolution of electronic states during the "breaking and making" of molecular bonds without the need of spectroscopic information as in a typical "femto-chemistry" experiment in the optical regime [77].

Decades of studies for ion impact, with intense optical lasers as well as with synchrotron radiation established dedicated experimental tools for investigations of multiple ionization and fragmentation processes in atoms and molecules. Starting with recording total ionization cross sections and intensity-dependent ion yields respectively, and continuing with measurements of singly or multiply differential cross sections, instrumental progress recently culminated in experiments yielding fully differential data for ionization and fragmentation of simple atomic [19,78-82] and molecular [83-86] systems. Here, a breakthrough was achieved with the invention of coincident cold-target recoil-ion and electron momentum spectroscopy. These spectrometers (sometimes called "reaction microscopes", REMI [87-89]), based on combined electric- and magnetic-field projection techniques, time- and position-sensitive micro-channel plate (MCP) detectors and supersonic gas jets providing cold atomic or molecular targets, allowed for the simultaneous and coincident determination of the full three-dimensional (3D) momentum vectors for several charged reaction fragments (the socalled kinematically complete experiments). Moreover, due to the fact that emerging fragments are imaged with a large (often reaching $100 \%$ of $4 \pi$ ) solid angle, in many cases these machines enabled recording comprehensive probability densities of the final manyparticle momentum states. This, along with the availability of reliable background elimination procedures exploiting momentum conservation conditions [90,91], which generate unprecedentedly clean spectra, made this technique extremely successful in timeresolved experiments [73,74,92-97]. Because of its capabilities to separate different reaction channels leading to the same final states and cleanly reveal even weak ionization pathways based on the energy- and angular-resolved many-particle detection, this approach has now 
become the method of choice and fore-front state-of-the-art technique for atomic and molecular research with FELs [38,39, 43-48] and HHG [73,74], as well as in collision physics $[78,79,89]$.

The main goal of the present contribution is twofold. First, we will provide a brief overview of experiments on few-photon-induced double and multiple ionization of atoms and molecules performed over the last five years at the first VUV-FEL in the world, the Freeelectron LASer at Hamburg (FLASH) employing a dedicated "Reaction Microscope". We will concentrate on distinguishing direct and sequential fragmentation pathways for these reactions in the photon energy range of $30-50 \mathrm{eV}$, that has received most attention, experimentally as well as theoretically and characterize their salient features by inspecting differential or multi-differential cross sections (note that several experiments of this kind have been performed at the EUV-FEL at the Spring 8 Compact SASE Source (SCSS) in Japan at energies below $25 \mathrm{eV}$ (see, e.g., [46,47]), and, very recently, at the first X-ray FEL, LINAC coherent light source (LCLS) at Stanford: however, they will not be subject of the present review). Second, by presenting the very first experimental results on time-resolved molecular dynamics involving the absorption of two or more EUV photons we envisage what we think is one of the most exciting future field in atomic and molecular physics at FELs: Infrared-, terahertz-, EUV or X-ray pump-probe experiments with few femtosecond timeresolution and any combination of the above wavelengths regimes for the pump or probe pulse, respectively, imaging emerging ionic fragments, photoelectrons and possibly scattered photons in multiple coincidence experiments.

The paper is organized as follows. In section 2 we briefly discuss the experimental apparatus, a dedicated "Reaction Microscope" focusing on the issues specific for the FEL experiments, and describe a split-mirror setup developed for EUV pump - EUV probe experiments mentioned above. Section 3 is devoted to the results on direct and sequential 
double ionization of $\mathrm{He}$ and $\mathrm{Ne}$ atoms upon two- or three-photon absorption, and Section 4 deals with multiple fragmentation of simple diatomic molecules, $\mathrm{D}_{2}$ and $\mathrm{N}_{2}$. In Section 5 we present and discuss first results of the EUV pump - EUV probe experiments on $\mathrm{N}_{2}$, and Section 6 gives a short summary of the manuscript as well as our view of the future perspectives.

\section{Experimental: "Reaction Microscope" for time-resolved studies at free-electron lasers}

\subsection{Dedicated "Reaction Microscope" for studies of atomic and molecular fragmentation at} FLASH

In several experimental campaigns at FLASH the Heidelberg "Reaction Microscope" (REMI), a multi-particle imaging spectrometer which allows measuring the 3D momentum vectors of several ions and electrons in coincidence, was used to explore non-linear reactions in atoms and small molecules. The sketch of the apparatus (including the split-mirror setup for time-resolved measurements described in Section 2.2) is shown in Fig. 1. In brief, the FLASH beam (typically 12 to 30 micro-bunches at $5 \mathrm{~Hz}$ repetition rate) was focused onto a collimated supersonic gas-jet target (propagating parallel to the FEL polarization direction) in the ultra-high vacuum chamber of the REMI. For single-pulse experiments described in Section 3 and 4 the focusing had been performed by the ellipsoidal grazing incidence mirror upstream along the FLASH beamline (BL2), providing a focus of $~ 15-20 \mu$ m diameter. For pump-probe experiments described in Section 5 the beam was refocused by a back-reflecting split-mirror described in Section 2.2 (configuration illustrated in Fig. 1) Created charged particles were projected onto two position-sensitive and multi-hit capable micro-channel plate (MCP) detectors (diameter $120 \mathrm{~mm}$ for ions, and $80 \mathrm{~mm}$ for electrons, position resolution 0.1 $\mathrm{mm}$, dead-time $10 \mathrm{~ns})$ by means of weak electric $(\sim 1 \mathrm{~V} / \mathrm{cm}$ for atomic and $\sim 40-50 \mathrm{~V} / \mathrm{cm}$ for molecular experiments) and magnetic (10 G) fields applied along the vertical axis 
(perpendicular to both, FEL propagation and polarization directions) achieving a detection solid-angle close to $4 \pi$ for all particles of interest. From the measured times of flight (TOF) and positions on the detector the initial momentum vectors of emerging ions and electrons were reconstructed.

The most prominent development for the dedicated FEL machine compared to the earlier generation of REMIs $[89,90]$ was further improvement of the background pressure in the spectrometer chamber $\left(<10^{-11}\right.$ mbar $)$ enforced by the severe vacuum requirements set by the desire to perform non-linear multiple ionization experiments in the EUV domain. In order to observe these reactions light intensities of order of $10^{13} \mathrm{~W} / \mathrm{cm}^{2}$ or higher are needed. Since the photon energies are typically high enough to efficiently ionize background atoms and molecules by a single photon with probabilities that exceed those for non-linear processes by at least two (often up to four) orders of magnitude, one encounters an enormous number of background signals not only from the focal region, but essentially all along the trace of the FEL beam through the spectrometer. At the same time one-photon single ionization of the target particles limits the effective target densities to $<10^{10}$ particles $/ \mathrm{cm}^{3}$ resulting in just a few, often less than one doubly or multiply charged ion of interest per shot. The upper limit of the target density is given by the detector saturation, space charge effects, and the requirements set by the coincidence measurements. Here one has to identify, e.g. by applying momentum conservation, those electronic and ionic fragments that emerged from the same atom or molecule in the target which becomes exceedingly difficult if more than a few events are detected per FEL shot. Although most of the above concerns are in principle also present in any experiment with intense optical lasers, still the background problem is typically much less severe in this case since ionization of background atoms or molecules due to its multiphoton nonlinear character is limited in space to the focal region, with only slightly larger effective volume compared to the reaction of interest. 
Fig. 2 illustrates the scheme of the coincident measurement and the performance of the REMI for a typical double ionization experiment. In Fig. 2a the measured longitudinal (parallel to the TOF axis) momentum component of $\mathrm{Ne}^{2+}$ ions created by the absorption of two $44 \mathrm{eV}$ FLASH photons is shown as a function of the sum-momentum of two detected electrons. Since the two absorbed photons carry negligibly small momenta, the final-state recoil-ion momentum is balanced by the sum-momentum of the electrons, which is reflected in the line observed in Fig. 2a. The sum-momentum of all three fragments (shown in Fig. 2b) is then close to zero and the width of the distribution for each of the three dimensions provides a sound quantitative estimate of the resolution achieved in the three-particle coincident experiment.

\subsection{Split mirror arrangement for the pump-probe experiments}

For the pump-probe experiments (Section 5) the REMI was attached to the unfocused FLASH beamline (BL3), and the FLASH beam after passing through the reaction chamber had been refocused on the target by a back-reflecting, narrow-band multilayer spherical mirror under normal incidence ( 1 inch $\mathrm{Mo} / \mathrm{Si}$ mirror, focal length $50 \mathrm{~cm}$, estimated focus diameter less than $10 \mu \mathrm{m})$. The mirror was cut horizontally into two identical half-spheres which can be moved relative to each other in order to produce two replicas of the FEL pulse. While the lower part of the mirror is mounted on a high-precision piezo-stage movable along the FEL propagation direction and allowing one to adjust the delay between both pulses in a range of \pm 2.5 ps with less then 100 as step resolution, the upper half sits on the tip-tilt stage enabling 2D relative adjustment of the reflections from both half-mirrors in the plane perpendicular to the beam propagation direction. Various mirrors from different sources (FOM institute AMOL, Amsterdam; Fraunhofer IOF, Jena; LBNL, Berkeley) have been used in experiments with different wavelength with reflectivity ranging from $30 \%$ to $60 \%$. 
The overlap of two focal points was initially adjusted observing an optical interference pattern obtained with a HeNe laser aligned along the beamline with $\sim 100 \mu \mathrm{m}$ precision. Further adjustment has been performed using a spatial imaging system for the created ions. Here, a strongly inhomogeneous electric field was created in the reaction point by setting a sharp metal needle introduced in the spectrometer on a high voltage magnifying the spatial image of the interaction (focal) point on the detector. By illuminating one or the other half of the mirror only, the vertical and horizontal positions of each of the two focal points were measured separately by the TOF and 2D position on the detector, respectively. Tilting and tipping one of the mirrors until TOFs and 2D positions coincide yielded an overlap between the two foci within about $20 \mu \mathrm{m}$. Fine tuning of the spatial overlap has been performed using non-linear $\mathrm{Ne}^{2+}$ signal. The time zero point $\mathrm{t}_{0}$ (exact temporal overlap of both pulses) in the first experiments has been verified by a sharp maximum observed in the measured delaydependent yield of multiple ionization of $\mathrm{N}_{2}$. The relative intensity of both pulse replicas can be chosen at will by varying the relative illumination of both mirror parts by the incoming FEL light.

\section{Double ionization of He and Ne atoms}

\subsection{Direct and sequential channels in EUV double ionization}

The most basic non-linear light-matter reaction involving more than one electron is represented by two-photon double ionization (TPDI) of the He atom. Due to its fundamental nature this process has received enormous theoretical attention (see e.g. [49-51, 53-57, 62-68, 98-102]) as soon as a possibility of its experimental realization appeared on the horizon. Surprisingly, even for the moderate intensities investigated, where second-order perturbative approaches are expected to be valid, the calculated total cross-sections for TPDI of He ( $\left.\sigma_{\text {TPDI }}\right)$ still vary over more than one order of magnitude [66]. Two recent measurements of $\sigma_{\mathrm{TPDI}}$ 
recently performed for photon energies of 41.8 [29,30] and $42.8 \mathrm{eV}$ [36] employing HHG and FLASH radiation, respectively, did not really solve the problem, mainly due to the fact that neither the pulse profile nor the pulse duration have been precisely known, and, thus, the estimated experimental intensity exhibits relatively large error bars such that the experiment can hardly discriminate between different theoretical predictions.

Two channels are usually distinguished in TPDI. As illustrated in Fig. 3, the "sequential double ionization" (SDI) is allowed if the photon energy $\hbar \omega$ is larger than the ionization potential $I_{P}^{+}$of the singly charged ion. It is usually seen as proceeding sequentially in time with two independent photo absorption events, one from the atom and one from the (relaxed) singly charged ion. The direct, or "non-sequential" double ionization (NSDI) instead, the only possibility at lower photon energies $\left(I_{P}^{+}>\hbar \omega\right.$ but $2 \hbar \omega>I_{P}^{+}+I_{P}$ with $I_{P}$ and $I_{P}{ }^{+}$being the ionization potentials of the neutral and the ion, respectively), requires the "instantaneous" absorption of the two photons through an intermediate virtual state ("vs" in the Fig. 3). It is considered to be a highly correlated process, with a smooth energy partition between the two electrons at lower photon energies but with distinct features predicted when approaching the boarder for sequential ionization at $I_{P}^{+}=\hbar \omega$. In principle sequential mechanisms can be realized as well at these photon energies. However, the second ionization step would require direct two-photon absorption by the singly charged ion, and, thus, "SDI" becomes a stepwise three-photon process, which is expected to become a dominant channel at intensities higher than those needed for the direct TPDI [36,38,42,59,102].

Since the dominance of direct or sequential channels, apart from the intensity of the light, is defined by the interrelation between the photon energy and the atomic structure (ionization potentials), considerable theoretical efforts have been undertaken to extend calculations for EUV double ionization to other rare gas atoms, in particular to Ne [52,58-61,69]. The latter, 
often being experimentally the more convenient target than $\mathrm{He}[38,39,43]$, provides a good comparison for qualitative understanding of ionization mechanisms.

Low-order perturbation theory expects the yield of ions produced by n-photon absorption to be proportional to $I^{n}$, where $I$ is the radiation intensity. Both of the He experiments mentioned above $[29,30,36]$ revealed the dominance of the two-photon NS channel for double ionization at light intensities up to $10^{14} \mathrm{~W} / \mathrm{cm}^{2}$ reflected in the linear intensity dependence of the double-to-single ionization ratio (which is equivalent to the quadratic intensity dependence of the doubly-charged ion yield in case if single ionization is not saturated). However, e.g., double ionization of $\mathrm{Ne}$ at $42 \mathrm{eV}$, where sequential TPDI is energetically allowed, manifested the same intensity dependence [36]. In the same work the $\mathrm{Ne}^{2+} / \mathrm{Ne}^{1+}$ ratio at $38.8 \mathrm{eV}$, where sequential TPDI is energetically forbidden, exhibited a quadratic intensity dependence, indicating that three photons are involved in the ionization process, whereas in [38] (for $38.4 \mathrm{eV}$ and at somewhat lower intensities), a nearly quadratic intensity dependence of the $\mathrm{Ne}^{2+}$ yield has been observed up to intensities of $\sim 6 \times 10^{12}$ $\mathrm{W} / \mathrm{cm}^{2}$, the lowest value used in [36], and a dependence of $I^{2.6}$ was found at higher $I$. From this assessment of the present situation one can clearly see that, as was previously realized for the single-photon and the strong-field (multiphoton) regimes, measurements of the total cross-sections alone do not allow one to draw a definite conclusions concerning the physical mechanisms underlying the few-electron ionization dynamics, and, thus, differential data are required.

\subsection{Recoil ion momentum distributions for double ionization of He and Ne}

Fig. 4 depicts the first differential data for TPDI of He. Momentum distributions of the $\mathrm{He}^{2+}$ ions were obtained at a photon energy of $\hbar \omega=44 \mathrm{eV}$ (a) and $52 \mathrm{eV}$ (b). The data are shown in the plane defined by the jet propagation direction and the spectrometer axis, and are integrated over the third momentum component (the one parallel to the FEL beam 
propagation). The FEL intensity was estimated as $\mathrm{I} \approx 10^{14} \mathrm{~W} / \mathrm{cm}^{2}$. The distribution in Fig. 4a displays a distinct maximum at the origin with essentially all events lying well within the circle marking the maximum $\mathrm{He}^{2+}$ momentum upon two-photon absorption (realized if both electrons are emitted in the same direction with equal energies). This behavior clearly differs from the momentum spectra obtained for single ionization [39] as well as for doubleionization induced by a single photon with similar energy above the threshold (see [103]), which both manifest a dipole-like shape. Since for double ionization the momentum of the doubly-charged ion is balanced by the sum-momentum of both emitted electrons, the dominance of the events with very low ion momenta indicates that both electrons are preferentially emitted back-to-back into opposite hemispheres with similar energies, thus, widely compensating the momenta of each other. This is in striking contrast with the dynamics found in single-photon double ionization, where exact back-to-back emission along the polarization direction is forbidden by the dipole selection rules. Thus, since this limitation is lifted for the case of TPDI, the difference between the ion momentum distributions for these two processes might readily be understood qualitatively.

In contrast to the data of Fig. 4a, the spectrum at $52 \mathrm{eV}$ (Fig. 4b) exhibits two maxima at longitudinal momenta close to \pm 1 a.u.. This is in good qualitative agreement with the recent theoretical predictions illustrated in Fig. 5. According to a series of calculations $[51,56,57,63-$ $65,68,101]$, a change of the shape of the energy distribution between both electrons should be expected. As discussed, e.g., in [63], for the photon energies of 40-45 eV equal energy sharing between both emitted electrons is needed in order to overcome the attraction of the nucleus and promote both electrons into the continuum (see Fig. 5a, taken from [63]), whereas as the photon energy approaches the SDI threshold $(54.4 \mathrm{eV})$, the asymmetric energy sharing starts to dominate. (Here it should be noted that even though this general trend can be clearly seen in the various calculations, there are significantly different energy sharings 
predicted at specific photon energies by different theoretical approaches). In this case both electrons are still preferentially emitted back to back, but one of them receives most of the excess energy resulting in the existence of the maximum at non-zero values in the total recoil-ion momentum (Fig. 4b, 5b). This behavior has been considered as a signature of the SDI via a virtual intermediate state, and was dubbed "virtual sequential ionization" $[51,56,57]$. Indeed, the predicted spectrum shape starts to resemble the one predicted for the photon energies above the SDI threshold shown in Fig. 5c (taken from [39], see also [56,57]).

Here it should be noted that the fine structure of the "virtual sequential ionization" contribution in TPDI of He at 50-52 eV differs in various calculations, sometimes exhibiting four maxima (see Fig. 5b) [56,57,63,69]. Unfortunately the momentum resolution of the present experiment (limited, among other factors, by space charge effects) does not allow us to reliably discriminate between these theoretical predictions. Factors defining the experimental resolution, its influence on the outcome of the calculations as well as the comparison to results from various theoretical approaches are considered in details elsewhere [104]. In the future, with increased repetition rates of micro-bunches at FLASH one might come into the position to significantly reduce space charge effects and envision to record fully differential cross sections as ultimate benchmark for theory.

Within the photon energy range used and due to limited available FLASH beamtime, experiments on SDI of He could not be performed. However, this channel has been studied in details for Ne. Fig. 6 displays recoil-ion momentum distributions for double ionization of $\mathrm{Ne}$ at $38 \mathrm{eV}$ (a) and $44 \mathrm{eV}$ (b). As one can see from the sketch presented in Fig. 3, for Ne at a photon energy of $44 \mathrm{eV}$ one does enter the sequential regime for TPDI. This is reflected in the observed momentum spectrum (Fig 6b), which clearly exhibits a ring-like structure corresponding to the intuitive expectations for sequential TPDI. Here, both electrons are assumed to be most likely emitted independently along the polarization direction, one from 
the neutral atom and one from the ground state of the singly charged ion, each exhibiting a dipole-like shape in its momentum distribution. The recoil spectrum of the doubly charged ion should then reflect the convolution of these two dipole structures. Whereas for He one would expect a clear two-ring structure with outer and inner rings on both sides reflecting the situation where both electrons either go into the same or opposite hemispheres, respectively (see Fig. 5c), for Ne this structure is smeared out because of (i) less excess energy and different electron energies in the continuum (depending on the final state of the doubly charged ion, see Fig. 7); (ii) different emission patterns of both electrons with a $\beta$-parameter of the second between 0 and 0.5, i.e. close to isotropic emission [52]; (iii) somewhat lower experimental momentum resolution achievable for $\mathrm{Ne}$ compared to $\mathrm{He}$ because of the internal gas-jet temperature resulting in a broadening of the spectrum $(\Delta \mathrm{p} \sim 0.7$ a.u., see $[39,104])$.

Fig. 6a displays the momentum distribution of $\mathrm{Ne}^{2+}$ ions obtained at a photon energy of $\hbar \omega=38.4 \mathrm{eV}$ (at the intensities $\mathrm{I} \geq 2 \times 10^{13} \mathrm{~W} / \mathrm{cm}^{2}$, where doubly charged ion yields showed a $\mathrm{I}^{2.6}$ intensity dependence). Here, sequential TPDI is energetically forbidden, and the recoilion momentum spectrum clearly resembles the pattern observed in Fig 4a for He, indicating the dominance of the direct TPDI channel and dominating back-to-back electron emission. However, in contrast to Fig. 4a, where essentially all events are found within the circle denoting the maximum sum-momentum of both electrons upon absorption of two photons, in Fig. $6 \mathrm{a}$ a noticeable amount of counts lie outside this circle, indicating that the contribution of the three-photon channel, though not dominating, is not negligible under these experimental conditions. This qualitatively agrees with the slope of doubly-logarithmic intensity-dependent $\mathrm{Ne}^{2+}$ yield lying in-between two and three as observed in [38]. Here it should be noted that according to some theoretical predictions, an intensity-dependent transition between twophoton direct and three-photon sequential mechanisms is expected to be rather sharp $[59,102]$ and, thus, the potential observation of non-negligible contributions from both channels might 
seem surprising. One of the possible reasons for this, as well as for somewhat different slopes of the $\mathrm{Ne}^{2+}$ yield curves observed in [36] and [38] might originate from the role of the intermediate resonance for the second step of the sequential channel since there is a number of excited states in the $\mathrm{Ne}^{+}$ion around $38.4 \mathrm{eV}$. Resonant enhancement might lead to the fact that, depending on the exact photon energies, ionization caused by different parts of the rather broad (and fluctuating) FEL pulse spectrum proceeds predominantly via different pathways, and might also result in the deviation (lowering) of the slope of the intensity-dependent ion yield curve (note that measurements [36] and [38] have been performed at slightly different photon energies).

\subsection{Sequential TPDI of Ne at $44 \mathrm{eV}$ : A kinematically complete experiment}

Difficulties with the separation of different contributing pathways as discussed in the previous subsection would be readily resolved if coincident electron spectra would be available. Unfortunately, because of the low cross sections, limited amount of beamtime, and low effective repetition rate (single bunches) of FLASH at the time those experiments were carried out, no fully differential data could be acquired under conditions where direct TPDI dominates. However, we did succeed in collecting fully differential data for sequential TPDI of $\mathrm{Ne}$ at $44 \mathrm{eV} \mathrm{[43],} \mathrm{and} \mathrm{these} \mathrm{results} \mathrm{shed} \mathrm{new} \mathrm{light} \mathrm{on} \mathrm{this} \mathrm{seemingly} \mathrm{simple} \mathrm{process.}$

Initially [105] SDI was characterized by several typical features: First, the electrons are considered to be emitted independent of each other. Second, whenever sequential TPDI is energetically allowed, it is expected to dominate the total cross section. Third, the predicted photoelectron spectrum is characterized by two lines defined by energy conservation and the two ionization potentials. While the latter two expectations are in line with the results of recent more elaborate calculations $[65,106]$, the former one has been questioned, and angular correlation between the emitted electrons have been predicted [60] pointing to the subtle nature of the process. 
In Fig. 7-8 we present electron spectra for SDI of $\mathrm{Ne}$ at $44 \mathrm{eV}$. Note that all electrons measured here are detected in coincidence with $\mathrm{Ne}^{2+}$ ions and, thus, fulfill the momentum conservation condition illustrated in Fig. 2. Fig. 7a displays the measured coincident twoelectron energy distribution integrated over all emission angles (with the dotted line showing the total singly differential energy spectrum). Apart from a small contribution from false coincidences (indicated by the arrow), the correlated energy spectrum consists of four distinct peaks symmetric with respect to the main diagonal. For each pair of peaks the energy of the electron from the first ionization step is centered at $22.5 \mathrm{eV}$ (peak 1), which is identical to the measured energy of electrons from single ionization. The second electron either emerges with a kinetic energy of about $3 \mathrm{eV}$ (peak 2), or with nearly zero energy (peak 3), indicating that the $\mathrm{Ne}^{2+}$ ion is left in the ${ }^{3} \mathrm{P}_{0,1,2}$ state $\left(40.9 \mathrm{eV}\right.$ with respect to the $\mathrm{Ne}^{+}$ground state, the triplet fine structure is not resolved), or in the ${ }^{1} \mathrm{D}_{2}$ state $(44.1 \mathrm{eV})$, respectively. The ${ }^{1} \mathrm{~S}_{0}$ final state $(47.1 \mathrm{eV})$ is energetically not accessible for sequential TPDI. The contributions from direct TPDI, which would be distributed along the lines parallel to the second diagonal $[64,65]$, cannot be observed in the spectrum.

Fig. $7 \mathrm{~b}$ displays the $2 \mathrm{D}$ momentum distribution of the electrons originating from double ionization. One can clearly observe three structures (marked by the arrows) corresponding to the different electron energies of Fig.7a. The outer ring (1) corresponds to the $22.5 \mathrm{eV}$ electrons from the first ionization step, the middle one (2) reflect those with $\sim 3 \mathrm{eV}$ energy from the second step leading to the ${ }^{3} \mathrm{P}$ final state of $\mathrm{Ne}^{2+}$, and the spot close to zero (3) originates from second-step, low-energy electrons corresponding to the ${ }^{1} \mathrm{D}$ final state.

From the measured three momentum components for each of the electrons we reconstruct their emission angular distributions (see e.g. Fig. 8a for the second electron emitted with the $\mathrm{Ne}^{2+}$ left in the ${ }^{3} \mathrm{P}$ state) and extract angular asymmetry parameters $(\beta)$ from fits (e.g. the full red line in Fig. 8a) containing $\beta_{2}$ and $\beta_{4}$ asymmetry parameters as expected theoretically for 
a two-photon process. The resulting angular $\beta$ parameters obtained for the electrons from the first $\left({ }^{(1)} \beta\right.$ ) and the second $\left({ }^{(2)} \beta\right)$ second SDI steps are presented in Fig. 8b. Again, only the events where the $\mathrm{Ne}^{2+}$ ion is left in the ${ }^{3} \mathrm{P}$ final state are included.

In Fig. $8 \mathrm{~b}$ we compare the experimental $\beta$-values (open symbols) with the theoretical predictions. Solid and dashed lines represent the results of the multi-configurational HartreeFock (MCHF) and Dirac-Fock (MCDF) calculations, respectively [43,61]. For a direct comparison, the theoretical results were averaged over the unresolved ${ }^{3} \mathrm{P}_{0,1,2}$ final states of the $\mathrm{Ne}^{2+}$ ion (see [61]). The predicted state-selective ${ }^{(2)} \beta_{2}^{2+}$ parameters considerably deviate from each other. Our experimental value lies much closer to the MCHF result, which is slightly above the upper limit of the experimental error. Noticeably, recent calculation by Kheifets [52] (shown as a dashed-dotted line in Fig. 8b) predict an even lower value, thus, placing our experimental point exactly in-between these two theoretical curves. A similar behaviour can be observed for the ${ }^{(2)} \beta_{4}^{2+}$ parameter. Here, both MCHF and MCDF calculations yield almost identical values, again lying at the upper limit of the experimental error bar, whereas the results of [52] are close to the lower one.

Fig. 8b also shows ${ }^{(2)} \beta_{2}^{2+}$ and ${ }^{(2)} \beta_{4}^{2+}$ parameters obtained in the non-coincident experiment of Braune et al. at $47.5 \mathrm{eV}$ [107] (full symbols without error bars). Whereas the ${ }^{(2)} \beta_{2}^{2+}$ value agrees well with the prediction of the MCHF calculation, and, accounting for the expected wavelengths dependence, also with our experimental result, the one for ${ }^{(2)} \beta_{4}^{2+}$ is much lower than all three theoretical values. Correspondingly, it also considerably differs from the value obtained in our experiment. Since none of the three calculations predict a significant wavelength dependence for ${ }^{(2)} \beta_{4}^{2+}$ in this energy range, the reasons for this discrepancy remain unclear. 
The coincidence scheme used allows us not only to measure the electron angular distribution for the second ionization step, but also for the first one (which is not feasible in non-coincident photoelectron spectroscopy because these electrons have the same energy as those from single ionization). Since according to the theoretical expectations [61] both electrons in sequential TPDI are not emitted independently, this might be imprinted in the angular distribution of the first step electrons, which, thus, should differ from the one obtained for pure single ionization. We do observe some difference reflected in the non-zero value of the $\beta_{4}$ parameter for the first step $\left({ }^{(1)} \beta_{4}^{2+}\right)$, slightly outside the statistical error bar $\left({ }^{(1)} \beta_{4}^{2+}=-0.12 \pm 0.11\right.$, red data point in the inset of Fig. 6b). Analysis of this effect in the framework of the MCHF and MCDF approaches [43] yield non-zero values of ${ }^{(1)} \beta_{4}^{2+}=0.08$ and ${ }^{(1)} \beta_{4}^{2+}=0.06$, respectively (red full and dashed lines in the inset of Fig. 8b). Thus, even though the observed deviations from zero are weak, we did find a discrepancy between theory and experiment beyond the statistical experimental error.

Modifications of the angular distribution for the first step electrons compared to single ionization raises a very general question concerning the nature of sequential TPDI, since it appears rather counterintuitive that the second electron, which might be set free much later within the FEL pulse, influences the emission characteristics of the first one. This brings one back to the conclusion stated in the initial paper on the subject [105], claiming that "there is no way of distinguishing sequential from simultaneous", i.e., that the timing of the electron emission can not be revealed from the experimental data.

\section{Multiple fragmentation of simple molecules}

\subsection{Direct and sequential TPDI of $\mathrm{D}_{2}$}

For molecules, the absorption of a few EUV photons resulting in the ejection of two or more electrons typically leads to further disintegration (dissociation, Coulomb explosion) of 
the created molecular ions. Therefore, in order to reveal the basic mechanisms of lightmolecule interactions one has to consider (apart from the role of electron-electron correlation) such specific molecular issues as a role of nuclear motion on a time scale of the EUV pulse, the coupling between electronic and nuclear degrees of freedom beyond the BornOppenheimer approximation etc. A fundamental test ground for such studies is the hydrogen (or deuterium) molecule, which is the only molecular system being readily accessible for theory and beautiful experiments for single-photon induced double ionization have been reported recently $[108,109]$. Pulse durations of the novel FEL sources match characteristic time scales of both, vibrational motion and fast dissociation of these lightest molecules, making investigation of few-photon-induced fragmentation especially intriguing.

Here we consider fragmentation of the $\mathrm{D}_{2}$ molecule irradiated by intense FLASH pulses at $\hbar \omega=38 \mathrm{eV}$, focusing on tracing dominating mechanisms of TPDI. Similar to the atomic case, there are two basic TPDI pathways, direct and sequential. For the latter channel the photons are assumed to be absorbed via an intermediate stationary state of the ion (assumed to be the $1 \mathrm{~s} \sigma_{\mathrm{g}}$ ground state of $\mathrm{D}_{2}{ }^{+}$which is populated with a probability of about 95 $\%$ in the first absorption step) in two steps which might be separated in time, whereas for the former one both photons are absorbed simultaneously through a virtual intermediate state, implying that TPDI occurs close to the internuclear distance (R) of a neutral molecule. Thus, it appears natural to expect that the signatures of both TPDI pathways can be traced in the kinetic energy release (KER) of the Coulomb exploding molecule, which is related to the internuclear distance $\mathrm{R}$.

In order to gain insight into the various processes that might occur upon absorption of the first photon, we plot in Fig. 9a the KER spectrum for non-coincident $\mathrm{D}^{+}$fragments, reflecting dissociative channels of $\mathrm{D}_{2}{ }^{+}$. Only fragments emitted perpendicular to the 
polarization direction $\left(\theta=90^{0} \pm 5^{0}\right)$ are considered here. For comparison we also show theoretical ab initio results obtained using the time-dependent method developed in [110].

In excellent agreement with the $a b$ initio calculations and previous measurements for one photon single ionization [111] all relevant fragmentation pathways, like ground-state dissociation (maximum at $\left.\mathrm{E}_{\mathrm{KER}}=0\right)$, dissociation via the $2 \mathrm{p} \pi_{\mathrm{u}}$ channel $\left(\mathrm{E}_{\mathrm{KER}} \approx 9 \mathrm{eV}\right)$ as well as contributions and interferences involving the decay of doubly excited (Q2) states [109] are well resolved (Fig. 9a), providing a sound basis for the understanding of TPDI.

The KER-spectrum for coincident $\mathrm{D}^{+}+\mathrm{D}^{+}$fragments integrated over all emission angles is shown in Fig. 9b. Two clear peaks are visible in the experimental data, with the high energetic part at $18 \mathrm{eV}$ corresponding to a Franck-Condon transition from the molecular ground-state to the repulsive 1/R Coulomb state. Such high KER is expected for direct TPDI, however, sequential ionization with a very short time gap (much less than the Coulomb explosion time) between both absorption steps or with the absorption of the second photon at any later time when the created ionic wave-packet reaches its inner turning point may also contribute. The low KER region, however, is accessible via sequential ionization only enabling a quantitative separation of both pathways with the help of theory. Advanced manyparticle quantum calculations by Martin et al. (included in [48]) deliver absolute cross sections for the sequential part along with relative KER distributions for direct TPDI such that, in comparison with experiment, not only both pathways can be disentangled but, moreover, first conclusions on the relative importance of both processes can be obtained. Thus, coincident KER spectra indirectly encode the time in between the absorption of the two photons through the internal molecular dynamics, proving the concept sometimes referred to as "molecular clock".

\subsection{Multiple fragmentation of $\mathrm{N}_{2}$ molecule}


As a more complex example of a multi-electron reaction with a large variety of possible fragmentation pathways, we discuss here the break-up of a nitrogen molecule exposed to intense FEL pulses. $\mathrm{N}_{2}$ represents one of the simplest few-electron diatomic systems, and its interaction with light has been extensively studied experimentally as well as theoretically. A wealth of results has been reported for single-photon interactions (see, e.g., [86,112-119], fragmentation by intense laser fields [83,84], two-colour time-resolved studies [73], and highresolution electron impact coincidence measurements [120]. Recently, several experiments on multiple ionization of $\mathrm{N}_{2}$ by few EUV photons have been performed at FLASH and SCSS. In particular, total ionization cross sections [33,34], time-of-flight spectra [42], as well as energy and angular distributions [44-46] of the ionic fragments for different fragmentation pathways have been reported.

In Fig. 10 we sketch the potential curves of $\mathrm{N}_{2}$ reachable by few-photon absorption from the $\mathrm{N}_{2}$ ground state as a function of the internuclear distance $\mathrm{R}$, whereas Fig. 11 displays a photo-ion-photo-ion-coincidence (PIPICO) spectrum measured at FLASH at a photon energy of $44 \mathrm{eV}$. Here, the TOF spectrum of the first detected ionic fragment is plotted as a function of the TOF of the second one. True coincident events resulting from three different Coulomb explosion channels $\left(\mathrm{N}_{2}{ }^{2+} \rightarrow \mathrm{N}^{+}+\mathrm{N}^{+}, \mathrm{N}_{2}^{3+} \rightarrow \mathrm{N}^{2+}+\mathrm{N}^{+}\right.$and $\left.\mathrm{N}_{2}^{4+} \rightarrow \mathrm{N}^{2+}+\mathrm{N}^{2+}\right)$ can be clearly identified as hyperbolic lines reflecting momentum conservation, whereas false coincidences (fragments originating from two different molecules) appear as a diffuse background. Based on the measured ion kinetic energies and setting software conditions on these coincidence lines, we can separate different channels of $\mathrm{N}_{2}$ break-up, and reveal the number of photons involved in the fragmentation process by measuring the intensity dependence of the yield of the particular pathway. The linearity of the intensity scale was ensured by inspecting the $\mathrm{H}_{2}{ }^{+}$ ion yield originating from single-photon ionization, which manifests a slope of 1 in a doublelogarithmic representation (see $[38,44,45])$. 
The results of this analysis are presented in the inset of Fig.11, where we plot intensitydependent ion yields for particular $\mathrm{N}_{2}$ decay channels. Surprisingly, the slopes of the yield curves indicate that several pathways involve more photons than the minimum number needed to reach the corresponding final states. First, we find that not only dissociative molecular dications $\mathrm{N}_{2}^{2+} \rightarrow \mathrm{N}^{+}+\mathrm{N}^{+}$, but also bound doubly charged molecular ions $\mathrm{N}_{2}{ }^{2+}$ $\left(\mathrm{X}^{1} \Sigma_{\mathrm{g}}{ }^{+}\right)$are both predominantly created by two-photon absorption. While this can be easily understood for the dissociative states $A^{1} \Pi_{u}, d^{3} \Pi_{g}$, and $D^{1} \Sigma_{u}{ }^{+}$since most of them simply cannot be populated via one-photon absorption, this is certainly different for the $\mathrm{X}^{1} \Sigma_{\mathrm{g}}^{+}$state (43 $\mathrm{eV}$ above the neutral $\mathrm{N}_{2}$ ground state) which is clearly in reach for one $44 \mathrm{eV}$ photon (see Fig. 10). The need for two photons to be absorbed is most likely due to exceedingly small cross sections close to the single-photon double ionization threshold, while sequential twophoton double ionization involving, e.g., excited states of $\mathrm{N}_{2}^{+}$is expected to be relatively large [44]. Second, the asymmetric dissociative dication channel $\left(\mathrm{N}_{2}{ }^{2+} \rightarrow \mathrm{N}^{2+}+\mathrm{N}\right)$ is dominated by three-photon absorption even though only two photons would be needed. Here, a one-photon threshold for the $(\gamma, 2 \mathrm{e})$ reaction has been established recently at $55 \mathrm{eV}$ with a cross section of only $5 \times 10^{-21} \mathrm{~cm}^{2}$ at $\sim 10 \mathrm{eV}$ excess energy [117]. At $44 \mathrm{eV}$ this excess energy is just reachable by two-photon SDI via the bound $\mathrm{N}_{2}{ }^{+}$states or dissociative $\mathrm{F}$ band, predominantly populated via single-photon absorption [73], such that the cross section for the $(2 \gamma, 2 \mathrm{e})$ pathway is intuitively expected to be small as well, and a dominance of the threephoton channel does not seem unreasonable. Certainly surprising, however, is that as much as five photons within one light-pulse $(\sim 25 \mathrm{fs})$ need to be absorbed for the creation of dissociating $\mathrm{N}_{2}{ }^{3+}$ ions, even though relying on the potential curves given in [121] already two would be energetically enough for the non-sequential triple ionization, and three would allow to reach this final state via sequential pathway. 
Further insight into the fragmentation dynamics can be gained from the angular distributions of the detected ionic fragments. In Fig. 12 we present the measured angular distribution as a function of KER for all $\mathrm{N}^{+}$fragments (a), $\mathrm{N}^{+}$fragments detected in coincidence $\left(\mathrm{N}^{+}+\mathrm{N}^{+}\right.$channel, b), and all $\mathrm{N}^{2+}$ fragments (c). Whereas non-coincident dissociating fragments (predominantly created by one-photon absorption) preferentially emerge along the FEL polarization axis (Fig 12a), ions from the Coulomb explosion produced by two-photon absorption (Fig 12b) exhibit a clear maximum in the perpendicular direction for the $\mathrm{A}^{1} \Pi_{\mathrm{u}}$ and $\mathrm{d}^{3} \Pi_{\mathrm{g}}$ final states, and only the fragments in the $\mathrm{D}^{1} \Sigma_{\mathrm{u}}^{+}$state are predominantly ejected along the laser electric field. Taking into account that the FEL pulse duration is much shorter than the rotational period of the molecule, we thus found strong evidences that $\mathrm{A}^{1} \Pi_{\mathrm{u}}$ and $\mathrm{d}^{3} \Pi_{\mathrm{g}}$ states cannot be created via the dissociating F- or H-bands by the absorption of the second photon and are most likely reached through the bound $\mathrm{N}_{2}{ }^{+}$states.

Considering the angular distribution of the $\mathrm{N}^{2+}$ fragments in Fig 12c, we observe the lowenergy ones, which mostly result from the $\mathrm{N}_{2}{ }^{2+} \rightarrow \mathrm{N}^{2+}\left({ }^{2} \mathrm{P}\right)+\mathrm{N}\left({ }^{4} \mathrm{~S}\right)$ dissociation channel, emerging along the FEL polarization axis. Therefore, this pathway can proceed via both, dissociative and bound intermediate states of $\mathrm{N}_{2}{ }^{+}$. On the contrary, fragments with KER > 10 $\mathrm{eV}$ originating from the $\mathrm{N}^{2+}+\mathrm{N}^{+}$channel are emitted preferentially in the perpendicular direction. Thus, this pathway, involving in total 5 photons as discussed above, does not proceed through the dissociating $\mathrm{F}$ - or $\mathrm{H}$-bands of $\mathrm{N}_{2}{ }^{+}$, but rather via the bound states of the latter ion, and afterwards via one of the bound or dissociating states of $\mathrm{N}_{2}{ }^{2+}$, with the exclusion of the $\mathrm{D}^{1} \Sigma_{\mathrm{u}}{ }^{+}$state which decays along the FEL polarization. The above constraints on the possible intermediate states involved in the production of the highly-charged fragments will become crucial for the understanding of the time-dependent $\mathrm{N}_{2}$ dynamics discussed in the next section. 


\section{First results of EUV pump - EUV probe experiments}

Here we briefly discuss a proof-of-principle time-resolved study on molecular fragmentation which employs EUV FLASH photons for both initiating and probing molecular dynamics of interest. For this illustrative experiment the nitrogen molecule was chosen as a target, mainly because of its rather large multiphoton ionization cross-sections in the EUV domain, the detailed knowledge of the fragmentation dynamics upon single photon absorption and considerable amount of data on few-photon fragmentation of $\mathrm{N}_{2}$ collected recently with the FELs (see previous Section). Here we present results obtained at a photon energy $\hbar \omega=38.7$ $\mathrm{eV}$, where we observed the most pronounced delay-dependent effects. Experiments were performed with the split-mirror setup described in Section 2.2. For this particular experiment we reached an intensity of $\sim 10^{14} \mathrm{~W} / \mathrm{cm}^{2}$, considerably higher, than for the single pulse measurement on $\mathrm{N}_{2}$ at $44 \mathrm{eV}$ discussed above. This is clearly imprinted in the observed ion spectra. As one can see from Fig. 13, where we present a PIPICO spectrum for the pumpprobe experiment at $38.7 \mathrm{eV}$ (integrated over the delay range of $\pm 300 \mathrm{fs}$ ), we achieve sevenfold ionization of the molecule, which at this photon energy requires at least 10 photons (compare to Fig. 11, where the highest charge state was limited to 4).

Fig. 14 depicts the measured KER spectrum of the $\mathrm{N}^{2+}$ fragments as a function of the delay between two identical FLASH pulses. Since both replicas of the FEL pulse were identical, the spectrum is symmetric with respect to zero delay. Clear delay-dependent structures can be observed, proving the capability of the split-mirror setup as well as spatial and temporal overlap of both pulses.

A detailed analysis of the pump-probe data and underlining molecular dynamics is beyond the scope of the current contribution, and will be reported elsewhere. However, we briefly discuss here the salient features of the spectra, relying on the channel identification based on the KER measurements and momentum conservation conditions set on the 
coincident lines visible in Fig. 14. We mainly concentrate on the $\mathrm{N}^{2+}$ fragments presented in Fig. 14, where particular contributing pathways are marked. One has to note that, in general, there are two types of delay-dependent phenomena to be expected in this kind of experiment. First, there is some "real" time-dependent molecular dynamics (e.g., signatures of the motion of the nuclei reflected in the delay-dependent KER). Second, since for the delays where two pulses overlap, the peak intensity of the FEL pulse is higher than for two non-overlapping pulses resulting in a different molecular response. In particular, non-linear reactions requiring direct (non-sequential) photon absorption should be enhanced around zero delays, resulting in autocorrelation-like patterns. Both of these effects are clearly visible in Fig. 14. One can trace a long high-energy tail extending beyond $40 \mathrm{eV}$ KER at zero delay (indicated by the arrow), which originates from higher-order non-linear ionization. Furthermore, the spectrum clearly manifests a series of bands (descending from zero delays in both directions) for which the fragment energy decreases with increasing time. This behavior is typical for pump-probe experiments where a final state is represented by the Coulomb explosion curve: First, the pump pulse creates a dissociative state of the molecular ion, which gets further ionized by the delayed probe pulse during its course of fragmentation. The inter-nuclear distance growing with time leads to smaller and smaller Coulomb explosion energies.

Here it should be noted that we observe only a very weak delay dependence for the $\mathrm{N}^{+}+$ $\mathrm{N}^{+}$final-state channel. The most likely reason for this is that, as shown in Section 4.2 by the analysis of the fragment angular distribution (for a somewhat higher photon energy), most of these ions are not produced via sequential (i.e., delayed) ionization of the dissociating states of the $\mathrm{N}_{2}{ }^{+}$ion, but rather via its bound states and, therefore, a potential delay dependence would be restricted to signatures of the vibrational motion in the intermediate state. On the contrary, as discussed above, there is a variety of dissociating states of the dication $\mathrm{N}_{2}{ }^{2+}$ 
which might be involved in the production of higher charge states, and, thus, their dynamics can be probed by absorbing one or few photons from the probe pulse while dissociating.

In order to get more insight into the delay-dependent features of the spectrum in Fig. 14, in Fig 15 we plot individual KER spectra for different pathways leading to the production of $\mathrm{N}^{2+}$ ions at two different delays, zero, and 275 fs. For the sake of visual convenience, all the curves are normalized to 1 at their respective maxima. Fig. 15a displays a non-coincident spectrum, where all $\mathrm{N}^{2+}$ ions are included for both delays. For this plot it can be clearly seen that the signal at higher KER values is enhanced at small delays, whereas in the low-energy region additional structures appear at later times. Two peaks appearing at $\sim 3 \mathrm{eV}$, and $8 \mathrm{eV}$ clearly manifest themselves in the coincident $\mathrm{N}^{+}+\mathrm{N}^{2+}$ spectrum (Fig. 15b) reflecting, most likely, one-photon ionization of the dissociating $\mathrm{N}_{2}^{2+}$ dication. On the other hand, the highenergy part of the non-coincident spectrum in Fig. 15a is obviously formed by the contribution from higher charge states (Fig. 15c,d). E.g., the high energy tail visible in the 2D spectrum in Fig. 14 at zero delay originates mostly from the $\mathrm{N}^{2+}+\mathrm{N}^{3+}$ final state. For both, $\mathrm{N}^{2+}+\mathrm{N}^{2+}$ and $\mathrm{N}^{2+}+\mathrm{N}^{3+}$ channels the KER spectra at large delays overall extend further towards lower energies compared to the results for overlapping pulses, and also exhibit pronounced structures in the low-energy region indicating rich dissociation dynamics. However, reliable identification of the states involved would require more detailed analysis of the experimental data (e.g., angular distributions for particular pathways at different delays, intensity dependences of the ion yields for different final states etc.), as well as better knowledge of the relevant (high-lying) potential curves. Furthermore, for routine unambiguous separation of different fragmentation channels, coincident electron spectra would become an indispensable and necessary tool.

\section{Conclusions and outlook}


Free-electron lasers along with high-harmonic based attosecond sources opened a new era for studies of light-matter interactions expanding non-linear physics and time-resolved measurements into the EUV and X-ray domain. Among other advances, the availability of FLASH, the first FEL user facility, in combination with rapid development of multi-particle momentum imaging techniques enabled a series of pioneering experiments on atomic and molecular fragmentation. Starting by inspecting intensity-dependent ion yields, these studies yielded differential and, for certain cases, even kinematically complete data, and triggered enormous theoretical activity. In particular, recoil-ion momentum distributions for a prototype non-linear reaction, direct TPDI of He measured at two different photon energies, 44 and $52 \mathrm{eV}$, stimulated a series of calculations for this observable and showed good qualitative agreement with theory. Similar experiments on TPDI of $\mathrm{Ne}$ at $38 \mathrm{eV}$ helped to interpret the results of ion-yield measurements revealing the dominance of a direct channel, but also highlighted the need to measure coincident electron spectra. Fully differential data for sequential TPDI of $\mathrm{Ne}$ at $44 \mathrm{eV}$ demonstrated that even this, presumably "simple" reaction raises a number of intriguing issues, since some basic questions on two-electron emission, e.g., whether or not both electrons can be ejected in the same direction along the polarization axis remain unresolved theoretically [60,61]. For molecules, coincident momentum spectroscopy of ionic fragments along with detailed theoretical analysis enabled reasonable understanding of the Coulomb explosion dynamics following direct and sequential TPDI for simplest diatomic systems such as $\mathrm{D}_{2}$. A wealth of experimental results on larger molecules (apart from the $\mathrm{N}_{2}$ data discussed in section 4.2, we have also performed analogous measurement for $\mathrm{O}_{2}$ ) yielded a qualitative picture of the few-photon-induced fragmentation and set a challenge to extend theory of non-linear two- or few-electron ionization to these systems. 
Experimentally, the next important milestone would be reached with a kinematically complete measurement on TPDI of He, which would allow for a detailed comparison with numerous theories available. This experiment, technically extremely challenging because of the background and cross section issues discussed in Section 2.1, will come closer to reality with the increase of the effective FLASH repetition rate expected for the next user run. This update will also considerably facilitate recording of coincident electron spectra for molecular fragmentation, necessary for reliable separation and identification of different decay channels. We have recently performed the first step in this direction, collecting ion-electron coincident data for $\mathrm{N}_{2}$ fragmentation at $52 \mathrm{eV}$ at low FEL intensities.

Another important issue which has to be resolved for a rigorous quantitative comparison between theory and experiment is the need for a detailed knowledge of the FEL pulse structure. FEL pulses generated by the so-called self-amplified spontaneous emission (SASE) cannot be directly reconstructed from the measured frequency spectrum because of the stochastic nature of the SASE process, and, thus, neither the pulse shape nor its averaged duration known only from the measured spectra and the machine parameters, cannot be considered reliable. Recently, considerable efforts have been undertaken in order to directly measure temporal and coherence properties of the FLASH pulses via linear or intensity autocorrelation $[41,122,123]$ and cross-correlation [124] measurements.

Ultrashort duration of the FLASH pulses provides a basis for a variety of timeresolved measurement schemes. Several two-color studies employing optical [125-127] and terahertz $[124,128]$ radiation synchronized with EUV pulses have been recently reported, and the development of the beam-splitter setups $[122,123]$ as well as the split mirror arrangement described in Section 2.2 opens a way for EUV-pump EUV-probe experiments. First results on $\mathrm{N}_{2}$ fragmentation presented in Section 6 illustrate the rich potential of coincident momentum spectroscopy and "Reaction Microscopes" for this kind of studies. It should be 
noted that from the measured delay-dependent KER spectra for different pathways one can also extract autocorrelation-like traces with different degree of non-linearity, thus, characterizing the averaged FEL pulse duration in situ. Apart from the $\mathrm{N}_{2}$ data presented here, first measurements on time-resolved imaging of $\mathrm{D}_{2}$ vibrational motion [129], $\mathrm{O}_{2}$ fragmentation, and isomerization of the $\mathrm{C}_{2} \mathrm{H}_{2}$ dication have been recently performed at FLASH employing this setup. In the future, following the same approach complemented with coincident electron detection, we envision tracing in real time dynamics of highly-excited states, autoionization reactions, light-induced structural rearrangement of polyatomic molecules etc. Due to the availability of the external optical laser synchronized with FLASH all these experiments can be performed on aligned molecular samples [125].

Until very recently two operating FEL facilities, FLASH and the EUV-FEL at SCSS covered the photon energy range from 12 to $200 \mathrm{eV}$. With the commissioning of the Linac Coherent Light Source (LCLS) in 2009 and upcoming Japanese [130] and European [131] XFELs, multiphoton and time-resolved experiments discussed here can be extended deep into the X-ray domain. As mentioned in the Introduction, this will open the way for a number of novel imaging techniques, including the possibility of coherent diffraction imaging of nanoscale objects [132,133] and recording "molecular movies" of light-induced chemical reactions via time-resolved X-ray diffraction or photoelectron holography [76]. In order to meet the requirements for these and others experiments with X-ray FELs, a next-generation experimental end-station combining the "Reaction Microscope" described here or "velocity map imaging" [134] spectrometer for charged particle detection with two large-area, fastreadout energy-resolving pnCCD detectors for X-ray photons has been recently developed [135]. This machine has been successfully commissioned at LCLS end of 2009, enabling unique experiments on atomic, molecular and cluster fragmentation as well as on coherent diffractive imaging of clusters, nanocrystals [136] and viruses. With this kind of experimental 
setup, in the view of further developments expected at the LCLS (including generation of few-femtosecond pulses in a pump-probe arrangement $[28,137])$, the update of the FLASH facility and two other upcoming XFELs deeming on the horizon, we envisage in the nearest future a number of exciting time-resolved experiments in atomic, molecular and biophysics imaging emerging ionic fragments, photoelectrons, as well as scattered and fluorescent photons in multiple coincidence experiments.

\section{Acknowledgements}

This work was supported by the Max-Planck Initiative DESY FEL (MIDFEL) and the MaxPlanck Advanced Study Group (ASG) at the Center for Free Electron Laser Science (CFEL) at Hamburg. We are grateful to A. Belkacem and LBNL staff, J. Verhoeven, P. Johnsson and M. Vrakking from AMOLF as well as E. Louis and F. Bijkerk (FOM) for designing and providing the multi-layer mirrors. This work would not have been possible without the sustained and enthusiastic support by the DESY accelerator team and beam line scientists, especially R. Treusch, S. Düsterer and M. Gensch. Y.H.J. acknowledges support from DFG project nr. JI 110/2-2, E.P, J.F.P.T. O.H., M.L. and M.F.K. acknowledge support from the DFG via the Emmy-Noether program and the Cluster of Excellence: Munich Center for Advanced Photonics. We thank our collaborators from the University of Frankfurt, Tohoku University Sendai, GANIL, University of Crete, University of Missouri Rolla, KSU and LBNL. Finally, we appreciated theoretical help from F. Martin, A. Voitkiv, A. Kheifets, B. McCurdy, J. Feist, J. Burgdörfer, S. Fritzsche, N. Kabachnik as well as discussions with P. Lambropoulos, R. Shakeshaft, B. Piraux, K. Bartschat and many others.

\section{References}

[1] M. Göppert-Mayer, Ann. D. Physik 9, 273 (1931). 
[2] V. Hughes and L. Grabner, Phys. Rev. 79, 314 (1950).

[3] G.S. Voronov and N.B. Delone, Sov. Phys.-JETP Lett. 1, 66 (1965).

[4] L.V. Keldysh, Sov. Phys.-JETP 20, 1307 (1965).

[5] A.M. Perelomov, V.S. Popov and M.V. Terent'ev, Sov. Phys.-JETP 23, 924 (1966).

[6] A.M. Perelomov, V.S. Popov and M.V. Terent'ev, Sov. Phys.-JETP 24, 207 (1966).

[7] A.I. Nikishov and V.I. Ritus, Sov. Phys.-JETP 23, 162 (1966).

[8] V.V. Suran and I.P. Zapesochny, Sov. Phys.- Techn. Phys. Lett. 1, 420 (1975).

[9] R. Dörner, Th. Weber, M. Weckenbrock, A. Staudte, M. Hattass, H. Schmidt-Böcking, R. Moshammer and J. Ullrich, Adv. At. Mol. Opt. Phys. 48, 1 (2002).

[10] A. Becker, R. Dörner and R. Moshammer, J. Phys. B 38 S753 (2005).

[11] J. Briggs and V. Schmidt, J. Phys. B 33, R1 (2000).

[12] L. Avaldi and A. Huetz, J. Phys. B 38, 861 (2005).

[13] M.Yu. Kuchiev, Sov.Phys.- JETP Lett. 45, 404 (1987).

[14] P.B. Corkum, Phys. Rev. Lett. 71, 1994 (1993).

[15] A. l'Huillier, L.A. Lompre, G. Mainfray and C. Manus, Phys. Rev. Lett. 48, 1814 (1982).

[16] B. Walker, B. Sheehy, L.F. DiMauro, P. Agostini, K.J. Schafer, K.C. Kulander, Phys. Rev. Lett. 73, 1227 (1994).

[17] D.N. Fittinghoff, P.R. Bolton, B. Chang and K.C. Kulander, Phys. Rev. A 49, 2174 (1994).

[18] S. Larochelle, A. Talebpour and S.L. Chin, J. Phys. B 31, 1201 (1998).

[19] Y. Liu, S. Tschuch, A. Rudenko, M. Dürr, M. Siegel, U. Morgner, R. Moshammer, and J. Ullrich, Phys. Rev. Lett. 101, 053001 (2008). 
[20] A. Emmanouilidou and A. Staudte, Phys. Rev. A 80, 053415 (2009).

[21] F. Mauger, C. Chandre and T. Uzer, Phys. Rev. Lett. 102, 173002 (2009).

[22] S.L. Haan, Z.S. Smith, K.N. Shomsky, P.W. Plantinga and T.L. Atallah, Phys. Rev. A 81, 023409 (2010).

[23] P. Agostini and L.F. DiMauro, Rep. Prog. Phys. 67, 813 (2004).

[24] F. Krausz and M. Ivanov, Rev. Mod. Phys. 81, 163 (2009).

[25] J. Feldhaus, J. Arthur and J.B. Hastings, J. Phys. B 38, S799 (2005).

[26] W. Ackermann et al., Nature Photonics 1, 336 (2007).

[27] T. Shintake et al., Nature Photonics 2, 255 (2008).

[28] Y. Ding et al., Phys. Rev. Lett. 102, 254801 (2009).

[29] [7] Y. Nabekawa, H. Hasegawa, E.J. Takahashi and K. Midorikawa, Phys. Rev. Lett. 94, $043001(2005)$.

[30] H. Hasegawa et al., Phys. Rev. A 71, 023407 (2005).

[31] H. Wabnitz et al., Phys. Rev. Lett. 94, 023001 (2005).

[32] E.P. Benis et al., Phys. Rev. A 74, 051402(R) (2006).

[33] A.A. Sorokin et al., J. Phys. B 39, L299 (2006).

[34] A. Föhlisch et al., Phys. Rev. A 76, 013411 (2007).

[35] M. Nagasono et al., Phys. Rev. A 75, 051406(R) (2007.)

[36] A.A. Sorokin et al., Phys. Rev. A 75, 051402(R) (2007).

[37] A.A. Sorokin, S.V. Bobashev, T. Feigl, K. Tiedtke, H. Wabnitz and M. Richter, Phys.

Rev. Lett. 99, 213002 (2007).

[38] R. Moshammer et al., Phys. Rev. Lett. 98, 203001 (2007).

[39] A. Rudenko et al., Phys. Rev. Lett. 101, 073003 (2008).

[40] T. Sato et al., Appl. Phys. Lett. 92, 154103 (2008).

[41] R. Mitzner et al., Phys. Rev. A 80, 025402 (2009). 
[42] M. Richter et al., Phys. Rev. Lett. 102, 163002 (2009).

[43] M. Kurka et al., J. Phys. B 42, 141002 (2009).

[44] Y.H. Jiang et al., J. Phys. B 42, 134012 (2009).

[45] Y.H. Jiang et al., Phys. Rev. Lett. 102, 123002 (2009).

[46] H. Fukuzawa et al., J. Phys. B 42, 181001 (2009).

[47] K. Motomura et al., J. Phys. B 42, 221003 (2009).

[48] Y.H. Jiang et al., Phys. Rev. A 81, 021401(R) (2010).

[49] E. Foumouo, G.L. Kamta, G. Edah and B. Piraux, Phys. Rev. A 74, 063409 (2006).

[50] R. Shakeshaft, Phys. Rev. A 76, 063405 (2007).

[51] D.A. Horner et al., Phys. Rev. A 76, 030701(R) (2007).

[52] A.S. Kheifets, J. Phys. B 40, F313 (2007).

[53] L.A.A. Nikolopoulos and P. Lambropoulos, J. Phys. B 40, 1347 (2007).

[54] I.A. Ivanov and A.S. Kheifets, Phys. Rev. A 75, 033411 (2007).

[55] A.S. Kheifets, I.A. Ivanov and I. Bray, Phys. Rev. A 75, 024702 (2007).

[56] D.A. Horner et al., Phys. Rev. A 77, 030703(R) (2008).

[57] D.A. Horner et al., Phys. Rev. A 78, 043416 (2008).

[58] L. Hamonou, H.W. van der Hart, K.M. Dunseath and M. Terao-Dunseath, J. Phys. B 41, 015603 (2008).

[59] M.G. Makris et al., Phys. Rev. A 77, 023401 (2008).

[60] S. Fritzsche et al., J. Phys. B 41, 165601 (2008).

[61] A. Kheifets, J. Phys. B 42, 134016 (2009).

[62] P. Lambropoulos and L.A.A. Nikolopoulos, New J. Phys. 10, 025012 (2008).

[63] E. Foumouo et al., J. Phys. B 41, 051001 (2008).

[64] J. Feist et al., Phys. Rev. A 77, 043420 (2008).

[65] X. Guan et al., Phys. Rev. A 77, 043421 (2008). 
[66] P. Antoine et al., Phys. Rev. A 78, 023415 (2008).

[67] J. Feist et al., J. Phys. B 42, 134014 (2009).

[68] D.A. Horner, T.N. Rescigno, and C.W. McCurdy, Phys. Rev. A 81, 023410 (2010).

[69] L. Hamonou, M. Lysaght and H.W. van der Hart, J. Phys. B 43, 045601 (2008).

[70] M. Drescher et al., Science 291, 1923 (2001).

[71] E. Goulielmakis et al., Science 305, 1267 (2004).

[72] M. Uiberacker et al., Nature 446, 627 (2007).

[73] E. Gagnon et al., Science 317, 1374 (2007).

[74] A. Sandhu et al., Science 322, 1081(2008).

[75] A.M. Lindenberg et al., Science 308, 392 (2005).

[76] F. Krasniqi et al., Phys. Rev. A 81, 033411 (2010).

[77] A.H. Zewail, J. Phys. Chem. 104, 5660 (2000).

[78] R. Moshammer et al., Phys. Rev. Lett. 73, 3371 (1994)

[79] A. Dorn et al., Phys. Rev. Lett. 86, 3755 (2001)

[80] M. Weckenbrock et al., Phys. Rev. Lett. 92, 213002 (2004).

[81] A. Rudenko et al., Phys. Rev. Lett. 99, 263003 (2007).

[82] A. Knapp et al., Phys. Rev. Lett. 89, 033004 (2002).

[83] E. Eremina et al., Phys. Rev. Lett. 92, 173001 (2004).

[84] D. Zeidler et al., Phys. Rev. Lett. 95, 203003 (2005).

[85] D. Akoury et al., Science 318, 949 (2007).

[86] M. Schöffler et al., Science 320, 920 (2008).

[87] R. Moshammer, M. Unverzagt, W. Schmitt, J. Ullrich and H. Schmidt-Böcking, Nucl. Instr. Meth. B 108, 425 (1996).

[88] R. Dörner, V. Mergel, O. Jagutzki, L. Spielberger, J. Ullrich, R. Moshammer and H. Schmidt-Böcking, Phys. Rep. 330, 95 (2000). 
[89] J. Ullrich, R. Moshammer, A. Dorn, R. Dörner, L.Ph.H. Schmidt and H. Schmidt-

Böcking, Rep. Prog. Phys. 66, 1463 (2003).

[90] V.L.B. de Jesus, A. Rudenko, B. Feuerstein, K. Zrost, C.D. Schröter, R. Moshammer and J. Ullrich, J. Electron Spectrosc. Relat. Phenom. 141, 127 (2004).

[91] A. Rudenko, B. Feuerstein, K. Zrost, Th. Ergler, V.L.B. de Jesus, C. Dimopoulou, C. D. Schröter, R. Moshammer and J. Ullrich, J. Phys. B 38, 487 (2005).

[92] A.S. Alnaser et al., Phys. Rev. A 72, 030702(R) (2005).

[93] Th. Ergler, A. Rudenko, B. Feuerstein, K. Zrost, C.D. Schröter, R. Moshammer and J. Ullrich, Phys. Rev. Lett. 95, 093001 (2005).

[94] Th. Ergler, A. Rudenko, B. Feuerstein, K. Zrost, C.D. Schröter, R. Moshammer and J. Ullrich, Phys. Rev. Lett. 97, 193001 (2006).

[95] M. Meckel et al., Science 320, 1478 (2008).

[96] I.A. Bocharova, H. Mashiko, M. Magrakvelidze, D. Ray, P. Ranitovic, C.L. Cocke, and I.V. Litvinyuk, Phys. Rev. A 77, 053407 (2008).

[97] A. Rudenko, Th. Ergler, B. Feuerstein, K. Zrost, C.D. Schröter, R. Moshammer and J. Ullrich, Chem. Phys. 329, 193 (2006).

[98] L.A.A. Nikolopoulos and P. Lambropoulos, J. Phys. B 34, 545 (2001).

[99] J. Colgan and M. S. Pindzola, Phys. Rev. Lett. 88, 173002 (2002).

[100] L. Feng and H.W. van der Hart, J. Phys. B 36, L1 (2003).

[101] S.X. Hu, J. Colgan and L.A. Collins, J. Phys. B 38, L35 (2005).

[102] P. Lambropoulos, L.A.A. Nikolopoulos and M.G. Makris, Phys. Rev. A 72, 013410 (2005).

[103] H.P. Bräuning et al., J. Phys. B 30, L649 (1997), 315149 (1998)

[104] M. Kurka et al., New J. Phys., submitted.

[105] H. Bachau and P. Lambropoulos, Phys. Rev. A 44, R9 (1991). 
[106] S. Laulan and H. Bachau, Phys. Rev. A 68, 013409 (2003).

[107] M. Braune et al., 25th Int. Conf. on Atomic, Electronic and Photonic Collisions, Freiburg, Germany, Fr034 (2007).

[108] A. Lafosse et al.-, J. Phys. B 36, 4683 (2003)

[109] F. Martín et al., Science 315, 629 (2007)

[110] J.L. Sanz-Vicario, H. Bachau and F. Martín, Phys. Rev. A 73, 033410 (2006).

[111] K. Ito, R.I. Hall and M. Ukai, J. Chem. Phys. 104, 8449 (1996).

[112] B. Zimmerman et al., Nature Physics 4, 649 (2008).

[113] M. Ahmad et al., J. Phys. B 39, 3599 (2006)

[114] M J. Besnard, L Hellner, G. Dujardin and D. Winkoun, J. Chem. Phys. 881732 (1988).

[115] M. Gisselbrecht et al., Phys. Rev. Lett. 96, 153002 (2006).

[116] T.J. Reddish et al., Phys. Rev. Lett. 100, 193001 (2008).

[117] P. Franceschi, D. Ascenzi, P. Tosi, R. Thissen and J. Zabka, J. Chem. Phys. 126, 134310 (2007).

[118] Th. Weber et al., J. Phys. B 34, 3669 (2001).

[119] D. Rolles et al, Nature 437, 711 (2005).

[120] M. Lundqvist, D. Edvardsson, P. Baltzer and B. Wannberg, J. Phys. B 29, 1489 (1996).

[121] A.D. Bandrauk, D.G. Musaev and K. Morokuma, Phys. Rev. A 59, 4309 (1999).

[122] R. Mitzner et al., Optics Express 16, 19909 (2008).

[123] W.F. Schlotter et al., Optics Letters 35, 372 (2010).

[124] U. Fruehling et al., Nature Photonics 3, 523 (2009).

[125] P. Johnsson et al., J. Phys. B 42, 134017 (2009).

[126] M. Krikunova et al., New J. Phys. 11, 123019 (2009).

[127] A. Azima et al., Appl. Phys. Lett. 94, 144102 (2009).

[128] M. Gensch et al., Infrared Phys. Technol. 51, 423 (2008). 
[129] Y. Jiang et al., Phys. Rev. A 81, 051402 (R) (2010).

[130] SPring-8 Compact SASE Source Conceptual Design Report, ed. by T. Tanaka and T. Shintake, RIKEN (2005).

[131] XFEL Technical Design Report, ed. by M. Altarelli et al., DESY-2006-097 (2007).

[132] R. Neutze, R. Wouts, D. van der Spoel, E. Weckert and J. Hajdu, Nature 406, 752 (2000).

[133] H.N. Chapman et al., Nature Physics 2, 839 (2006).

[134] A.T. Eppink, D.H. Parker, Rev.Sci.Instrum. 68, 3477 (1997).

[135] L. Strüder et al., Nucl. Instr. and Meth. A 614, 483 (2010).

[136] H.N. Chapman et al., Science (submitted)

[137] P. Emma et al., Phys. Rev. Lett. 92, 074801 (2004). 


\section{Figure captions:}

Figure 1. Sketch of the experimental setup. FEL polarization axis is parallel to the supersonic gas jet propagation direction.

Figure 2. (a) Recoil-ion versus electron sum-momentum along the TOF direction measured for TPDI of Ne at $44 \mathrm{eV}$. (b) Sum-momentum of all three particles for the same reaction. For both (a) and (b) momentum conservation condition on two other momentum components has been set.

Figure 3. Schematic of direct and sequential ionization of $\mathrm{He}$ and $\mathrm{Ne}$ at 38 and $44 \mathrm{eV}$, and of the relevant energy levels. VS: virtual state. The dashed line indicates an intermediate excited state in $\mathrm{Ne}^{+}$.

Figure 4. Density plot of the measured recoil-ion momentum distributions for double ionization of $\mathrm{He}$ at $44 \mathrm{eV}$ (a) and $52 \mathrm{eV}$ (b). The arrow indicates the direction of the FLASH polarization. $\mathrm{P}_{\|}$denotes the momentum component parallel and $\mathrm{P}_{\perp}$ - perpendicular to the polarization direction. The data are integrated over the third momentum component (parallel to the FLASH beam propagation direction). White circles mark the maximum possible $\mathrm{He}^{2+}$ momentum after two-photon absorption. Note that in (b) the $30 \mu \mathrm{m}$ nozzle of the supersonic gas jet was cooled to $20 \mathrm{~K}$ in order to achieve optimum momentum resolution (see [104]). Figure 5. Density plot of the calculated recoil-ion momentum distributions for double ionization of $\mathrm{He}$ at $42 \mathrm{eV}(\mathrm{a}), 50 \mathrm{eV}$ (b) and $60 \mathrm{eV}$ (c). Circles in (a) and (b) mark the maximum possible $\mathrm{He}^{2+}$ momentum after two-photon absorption. (a), (b): from [63]. (c): from [39].

Figure 6. Same as Figure 4, but for $\mathrm{Ne}$ at $38 \mathrm{eV}$ (a) and $44 \mathrm{eV}$ (b). Inner and outer circles in (a) indicate the maximum possible $\mathrm{Ne}^{2+}$ momentum after two- and three-photon absorption, respectively. 
Figure 7. (a) Coincident two-electron energy spectrum for TPDI of Ne by $44 \mathrm{eV}$ FLASH photons. Dashed line shows singly differential electron energy spectrum. (b) 2D momentum distribution of electrons for the same reaction. The numbers in both panels mark electrons from the first (1) and second (2,3) sequential ionization steps. (2) and (3) correspond to the $\mathrm{Ne}^{2+}$ ion left in the ${ }^{3} \mathrm{P}$ and ${ }^{1} \mathrm{D}$ states, respectively.

Figure 8. (a) Measured (full circles) and fitted (solid red line) angular distribution of the second-step electrons in TPDI of Ne at $44 \mathrm{eV}$ (see text). (b) Measured and calculated angular asymmetry parameters ${ }^{(2)} \beta_{2}^{2+}$ (black) and ${ }^{(2)} \beta_{4}^{2+}$ (blue) as a function of the photon energy. Inset (red): ${ }^{(1)} \beta_{4}^{2+}$-parameter for the electrons from the first step of TPDI. Open symbols: present experiment [43]; full symbols: experiment [107]; solid lines: multi-configuration Hartree-Fock calculation [43,61]; dashed lines: multi-configuration Dirac-Fock calculation [43,61]; dashed-dotted lines: Hartree-Fock calculation [53].

Figure 9. (a) KER spectrum of non-coincident $\mathrm{D}^{+}$fragments at $38 \mathrm{eV}$ taken under an emission angle of $90^{\circ} \pm 5^{\circ}$ with respect to the light polarization axis. (b) KER spectrum measured for coincident $\mathrm{D}^{+}+\mathrm{D}^{+}$pairs in comparison with theory (see text).

Figure 10. Sketch of the relevant potential curves of the $\mathrm{N}_{2}$ molecule. Arrows 1 and 2 denote the states reachable with one $44 \mathrm{eV}$ photon from the ground state of the neutral molecule and the singly charged ion, respectively.

Figure 11. Photo-ion-photo-ion coincidence spectrum measured for $\mathrm{N}_{2}$ fragmentation at 44 $\mathrm{eV}$. The time-of-flight of first ion is plotted versus the time-of-flight of the second. Inset: ion yields as a function of the FEL intensity $I$ for the same experiment. Symbols and solid lines denote the present measurements and fits $\left(\log \mathrm{Y}=\mathrm{n} \log I+\log \sigma_{\mathrm{n}}\right)$, respectively.

Figure 12. Polar density plots of ion angular distribution differential in the KER for various fragmentation channels. $\theta$ and the radius represent the angle with respect to the FEL polarization axis (horizontal) and the KERs, respectively. Curves display the KER spectra 
integrated over all fragment emission angles. (a) non-coincident $\mathrm{N}^{+}$fragments. (b) $\mathrm{N}^{+}+\mathrm{N}^{+}$ coincidences. (c) non-coincident $\mathrm{N}^{2+}$ ions.

Figure 13. Photo-ion - photo-ion coincidence spectrum measured for $\mathrm{N}_{2}$ fragmentation by two identical FEL pulses in a pump-probe experiment. Both pulses have identical intensities of $\sim 10^{14} \mathrm{~W} / \mathrm{cm}^{2}$ (photon energy $38 \mathrm{eV}$ ). The data are integrated over all delays between the pump and the probe pulses.

Figure 14. Measured kinetic energy release of $\mathrm{N}^{2+}$ fragment ions emerging from highly ionized $\mathrm{N}_{2}$ molecules as function of the pump-probe delay for the same experimental conditions as in Fig. 13. The arrow indicates an autocorrelation-like trace resulting from the delay-dependent yield of the highly-charged fragments. White lines denote the contributions of particular final-state products identified employing the coincidence conditions of Fig. 13. Note that this is only a schematic indication since rather broad delay dependent bands for different channels partly overlap.

Figure 15. Measured KER spectra for the pump-probe delay of 0 (full squares) and $275 \mathrm{fs}$ (open circles). (a) all $\mathrm{N}^{2+}$ fragments; (b) $\mathrm{N}^{+}+\mathrm{N}^{2+}$ coincidences; (c) $\mathrm{N}^{2+}+\mathrm{N}^{2+}$ coincidences; (d) $\mathrm{N}^{3+}+\mathrm{N}^{2+}$ coincidences. 


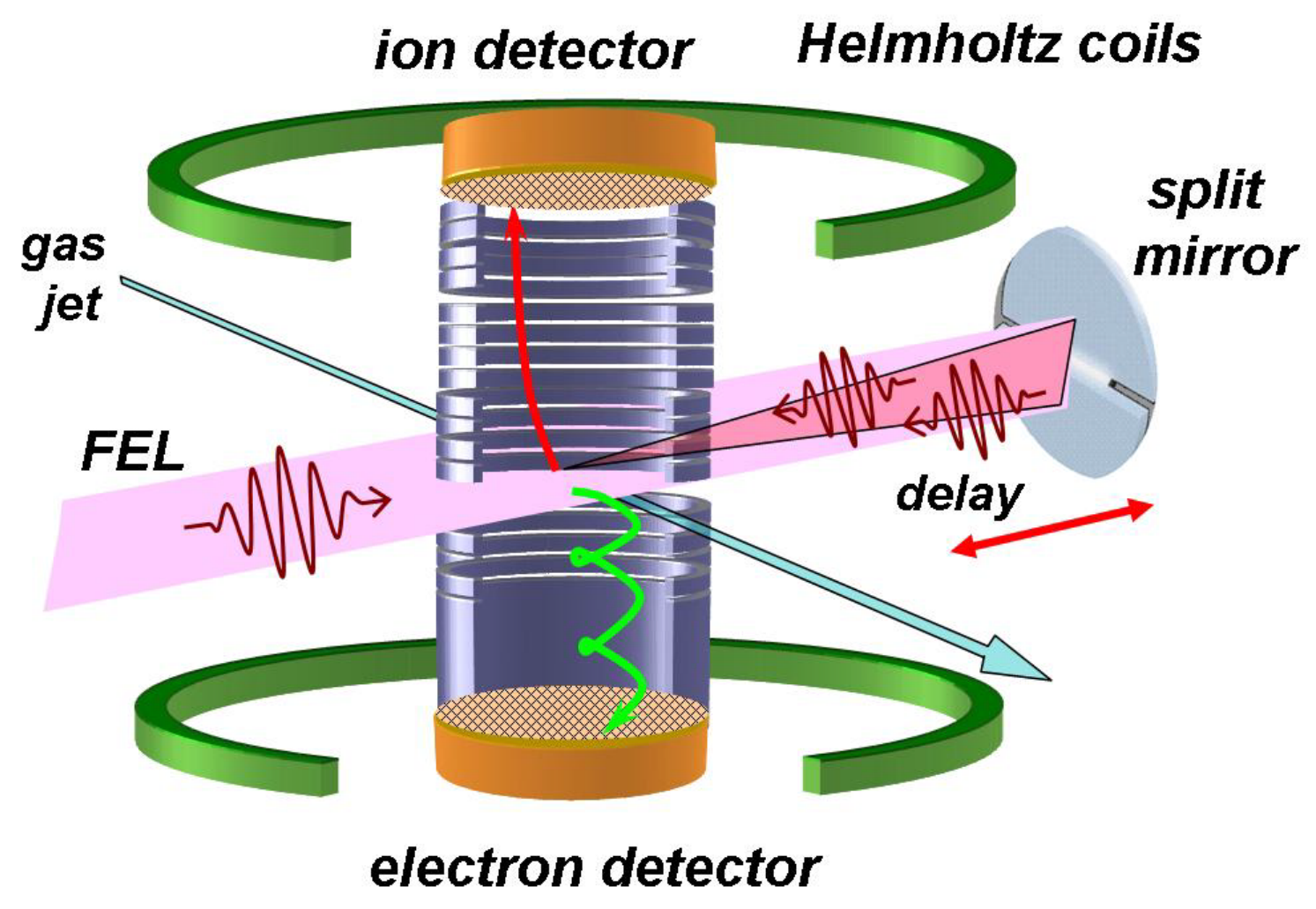

Figure 1 

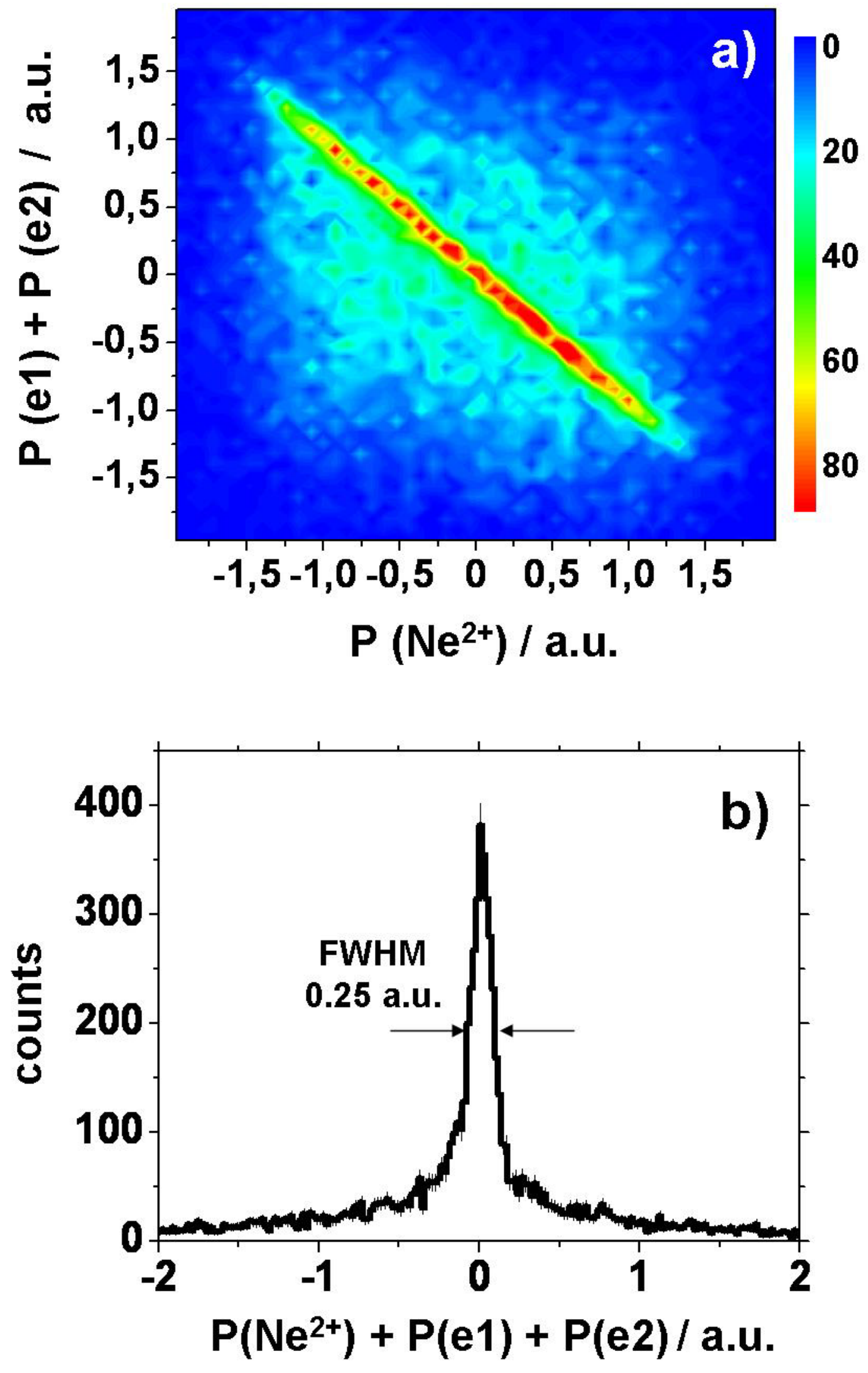

Figure 2 

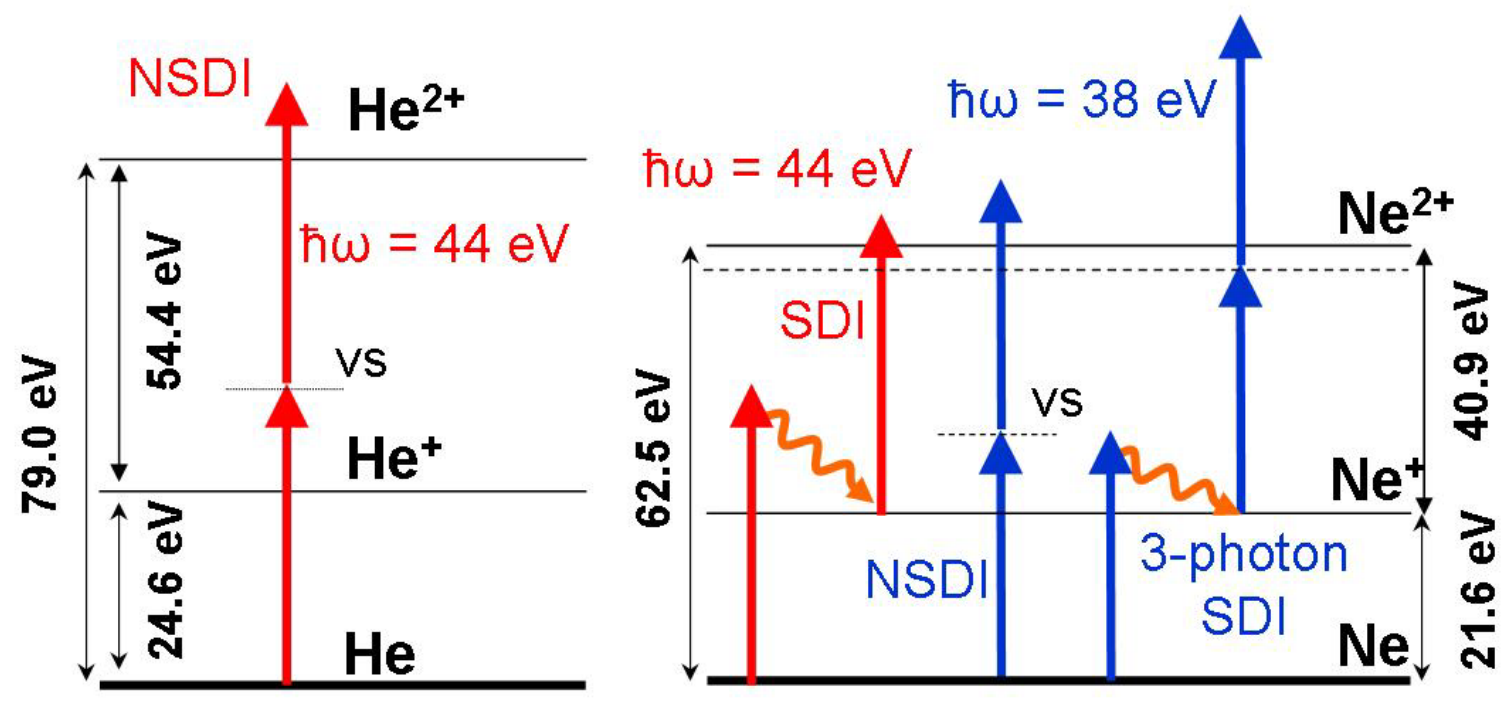

Figure 3 

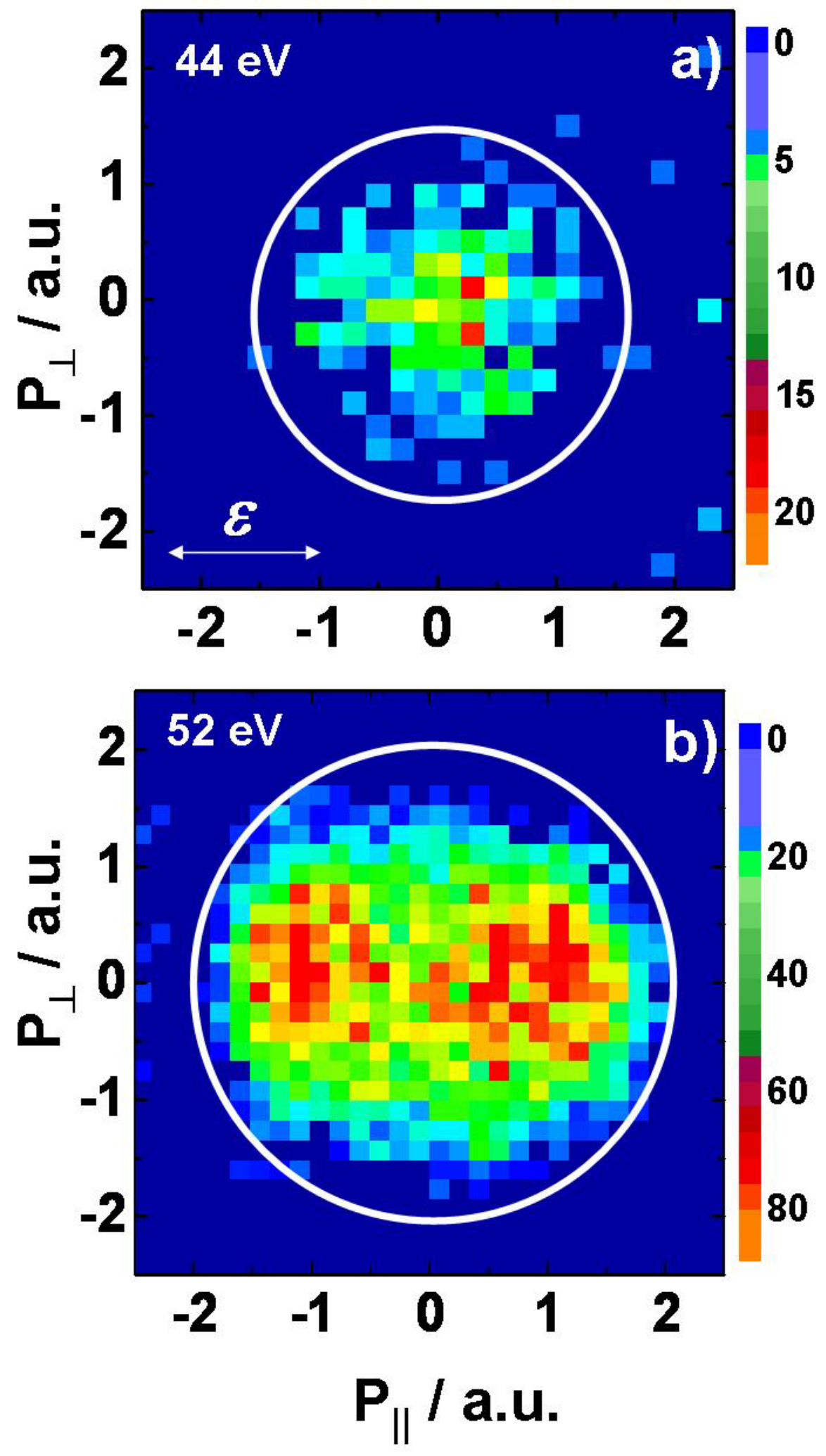

Figure 4 


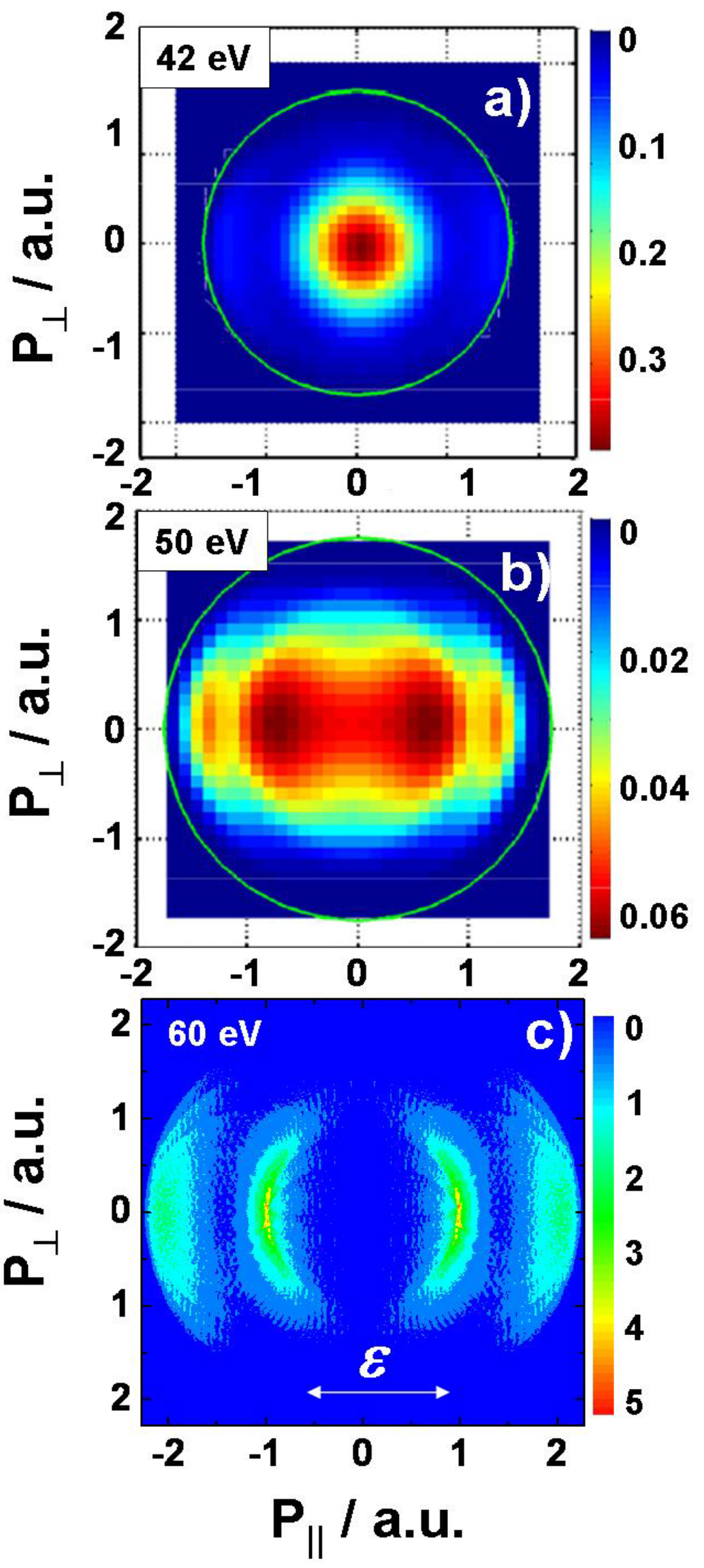

Figure 5 

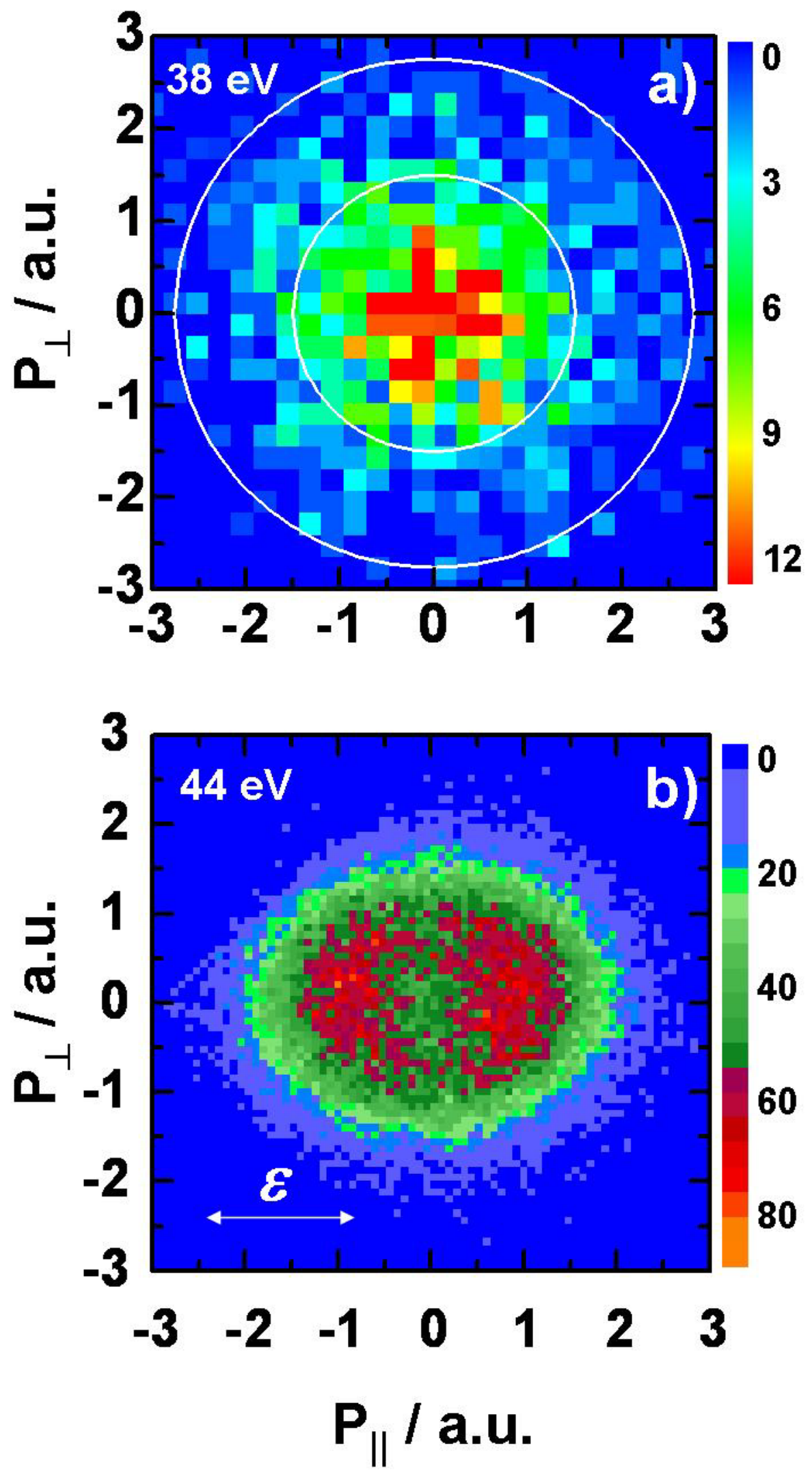

Figure 6 

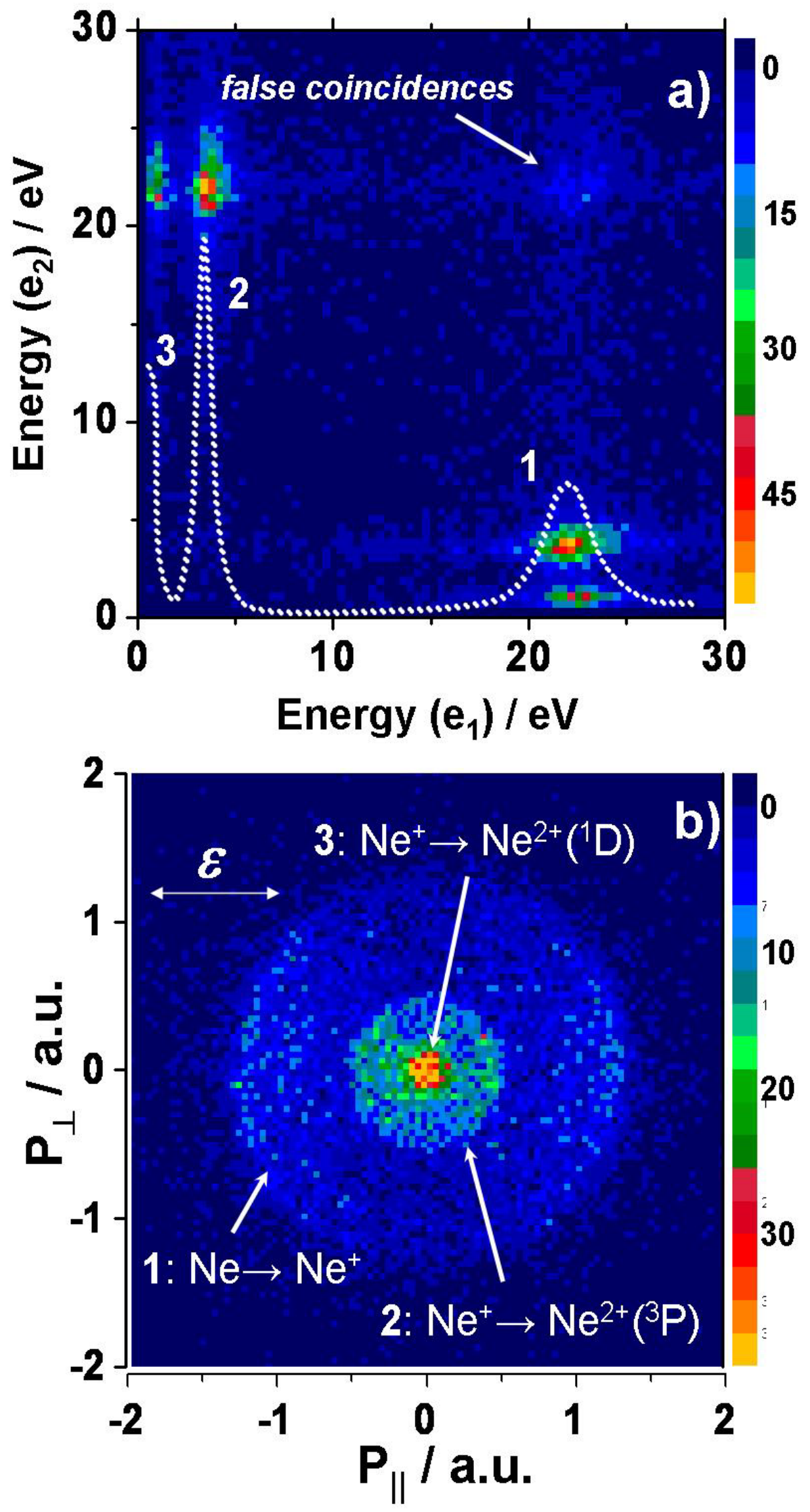

Figure 7 


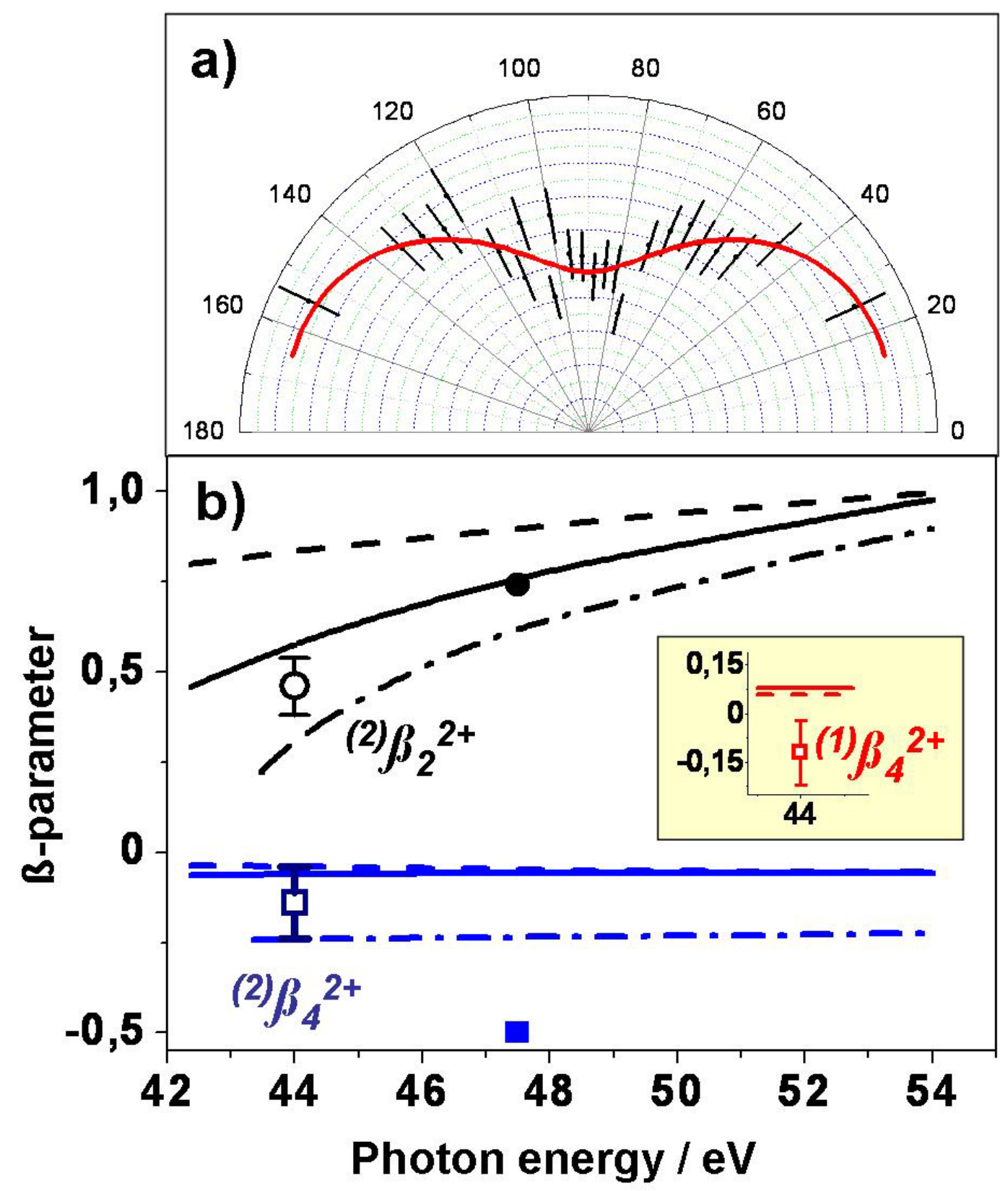

Figure 8 

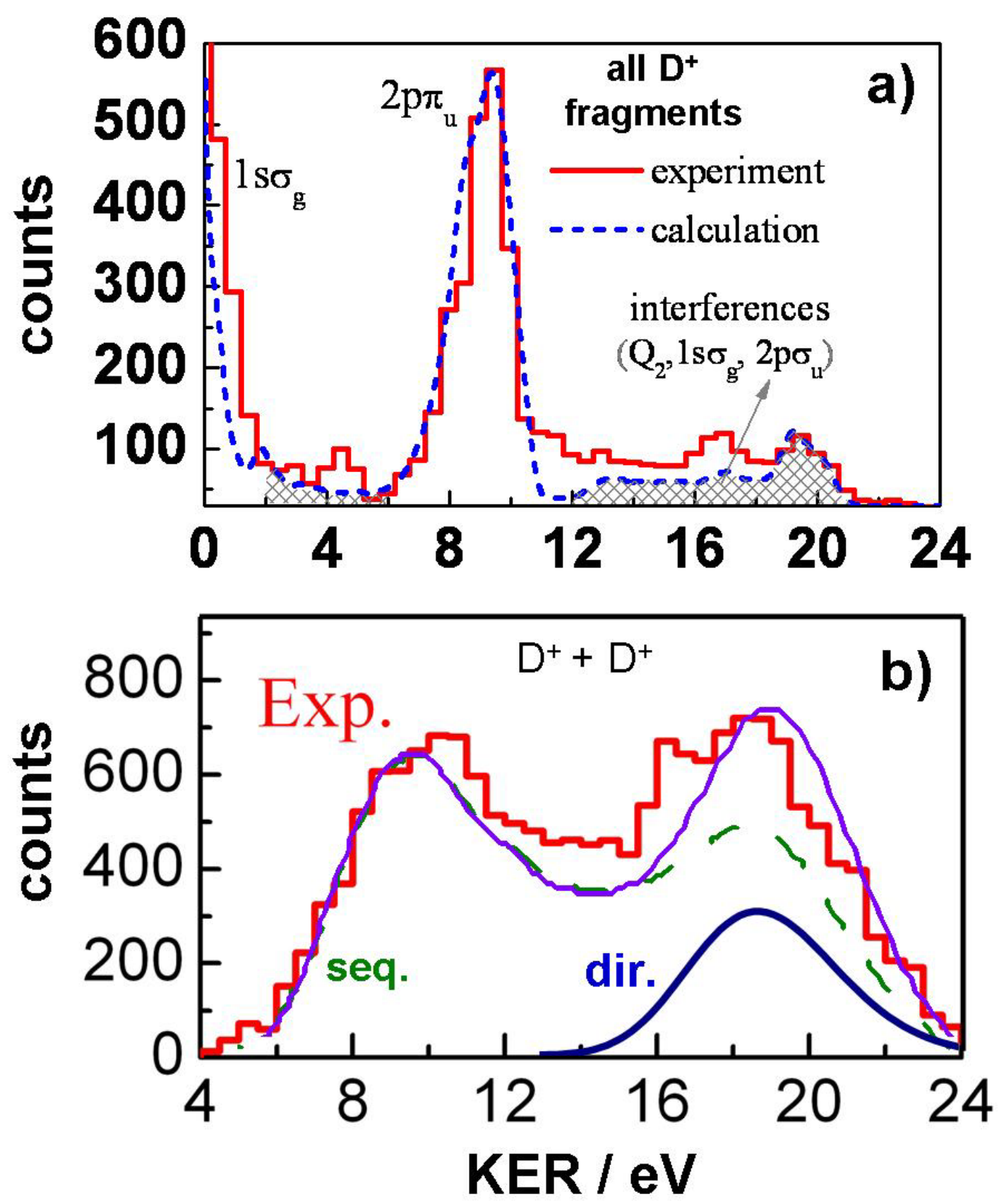

Figure 9 


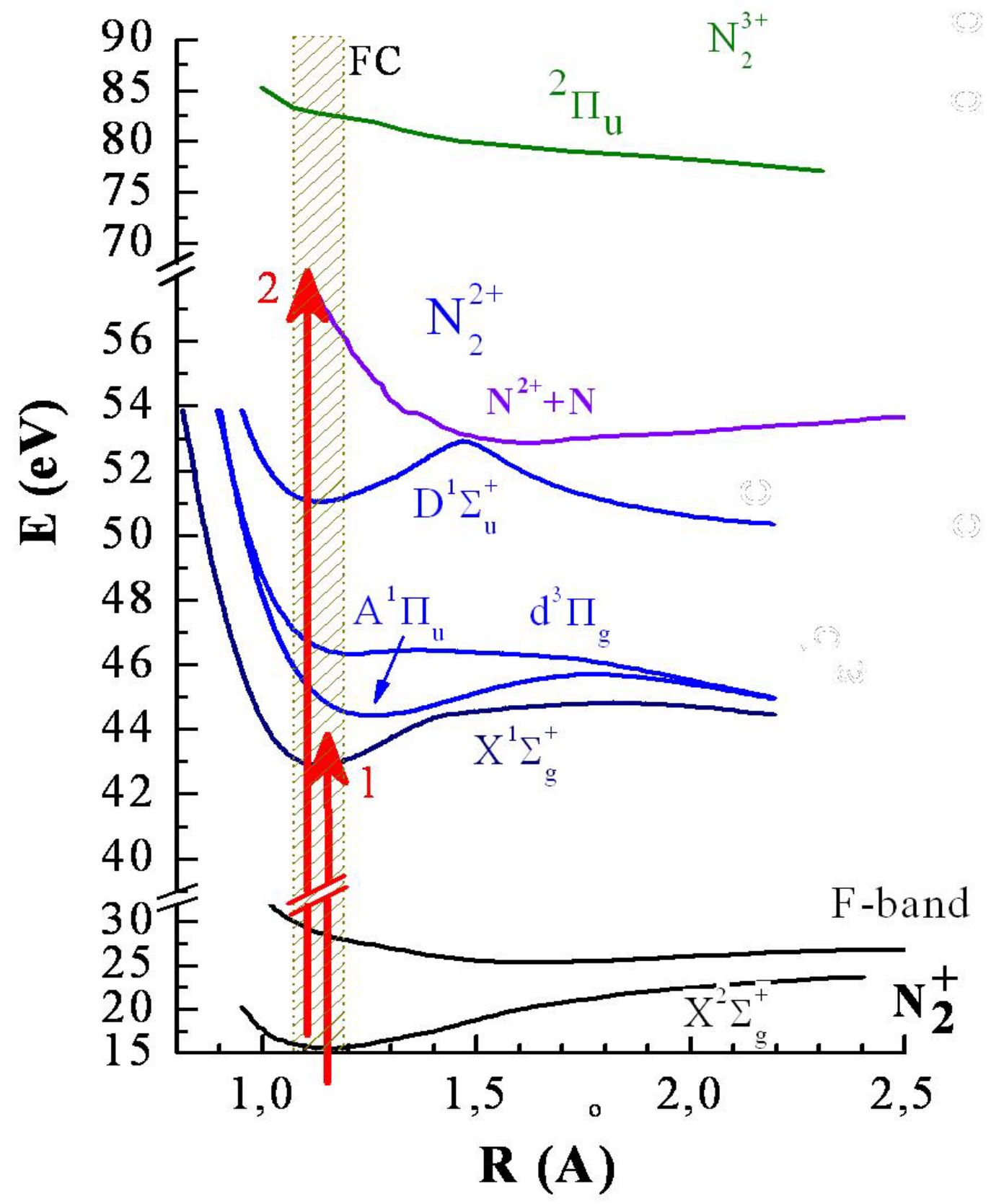

Figure 10 


\section{TOF $2 / \mu s$}

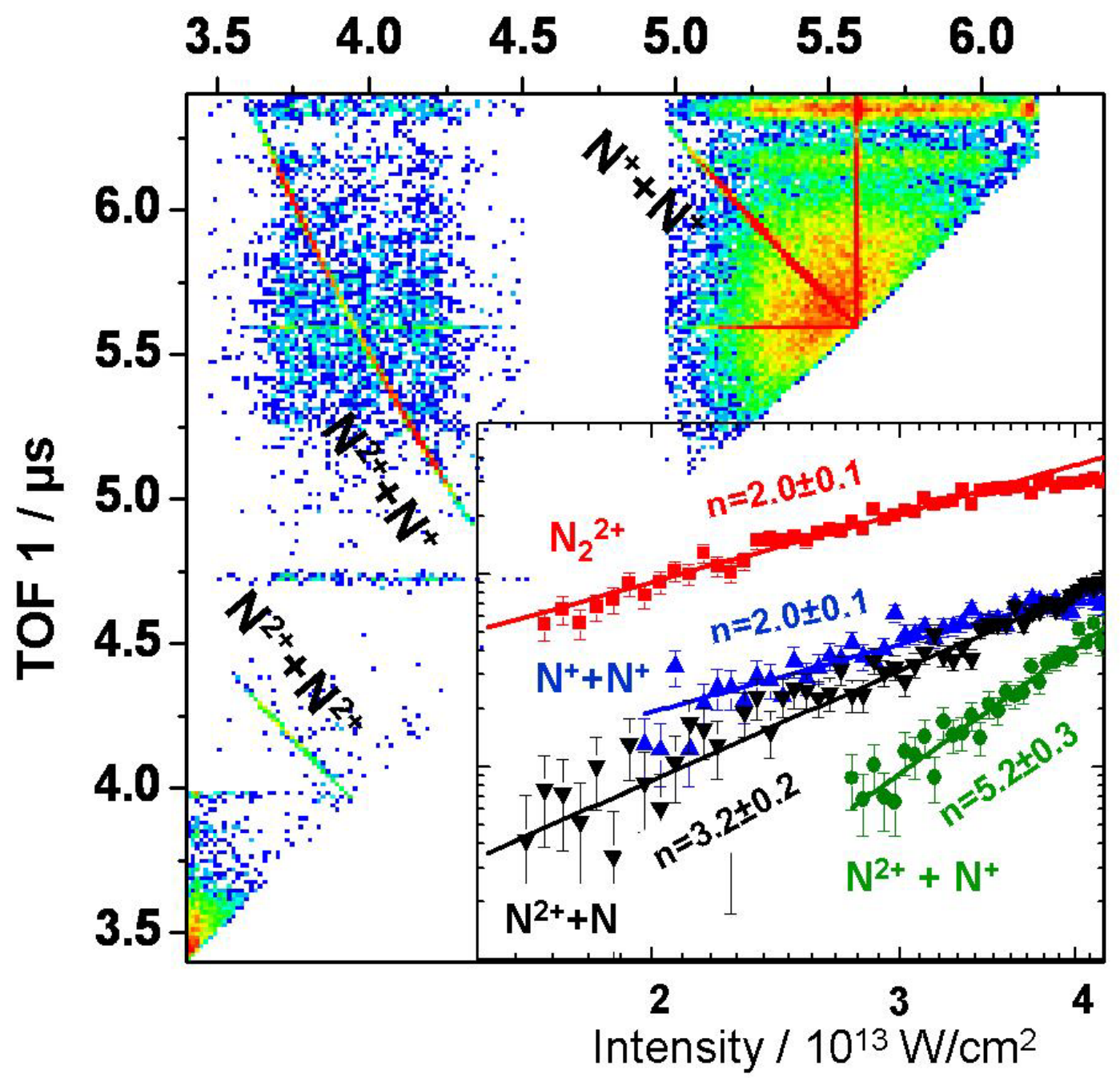

Figure 11 

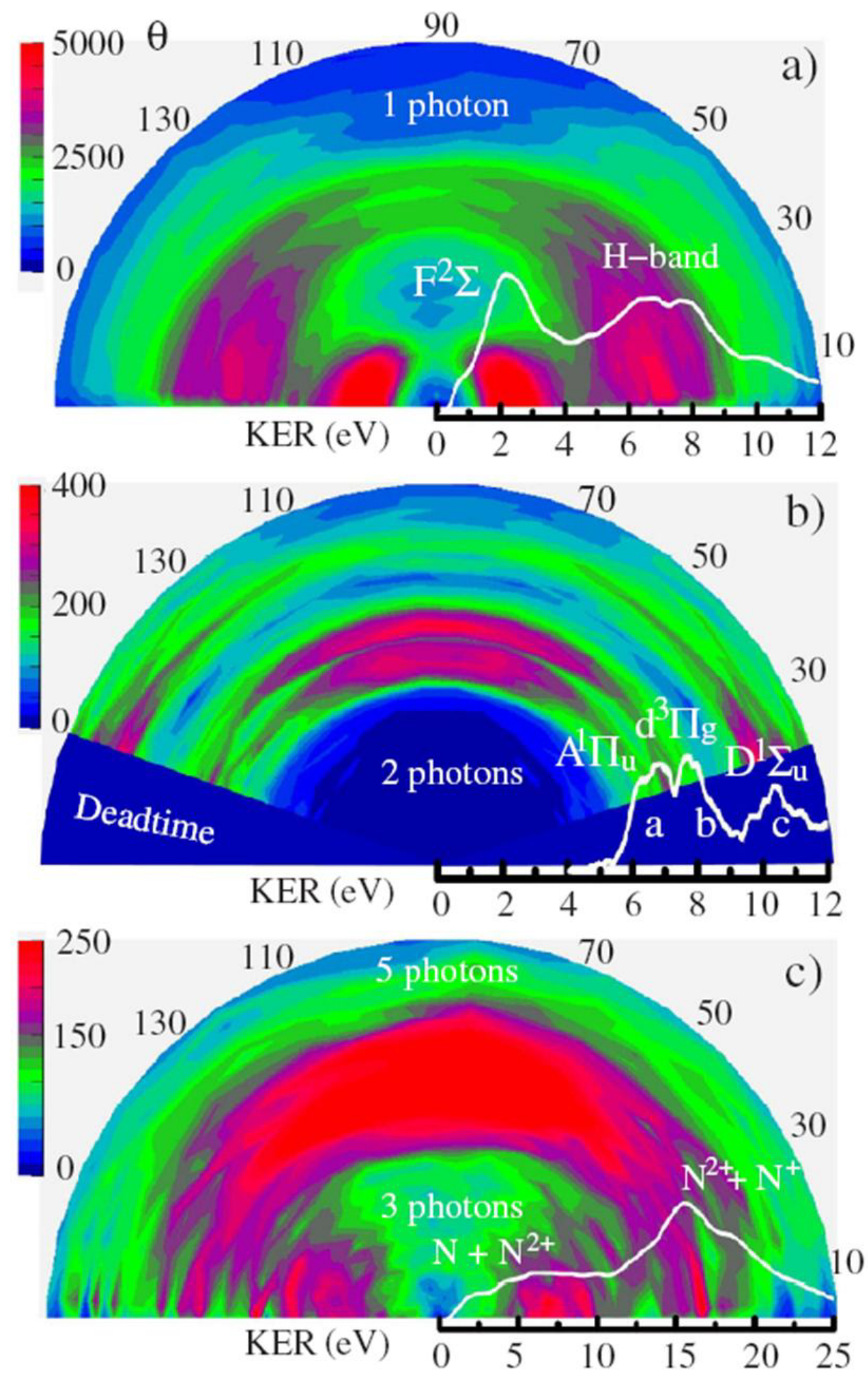

Figure 12 


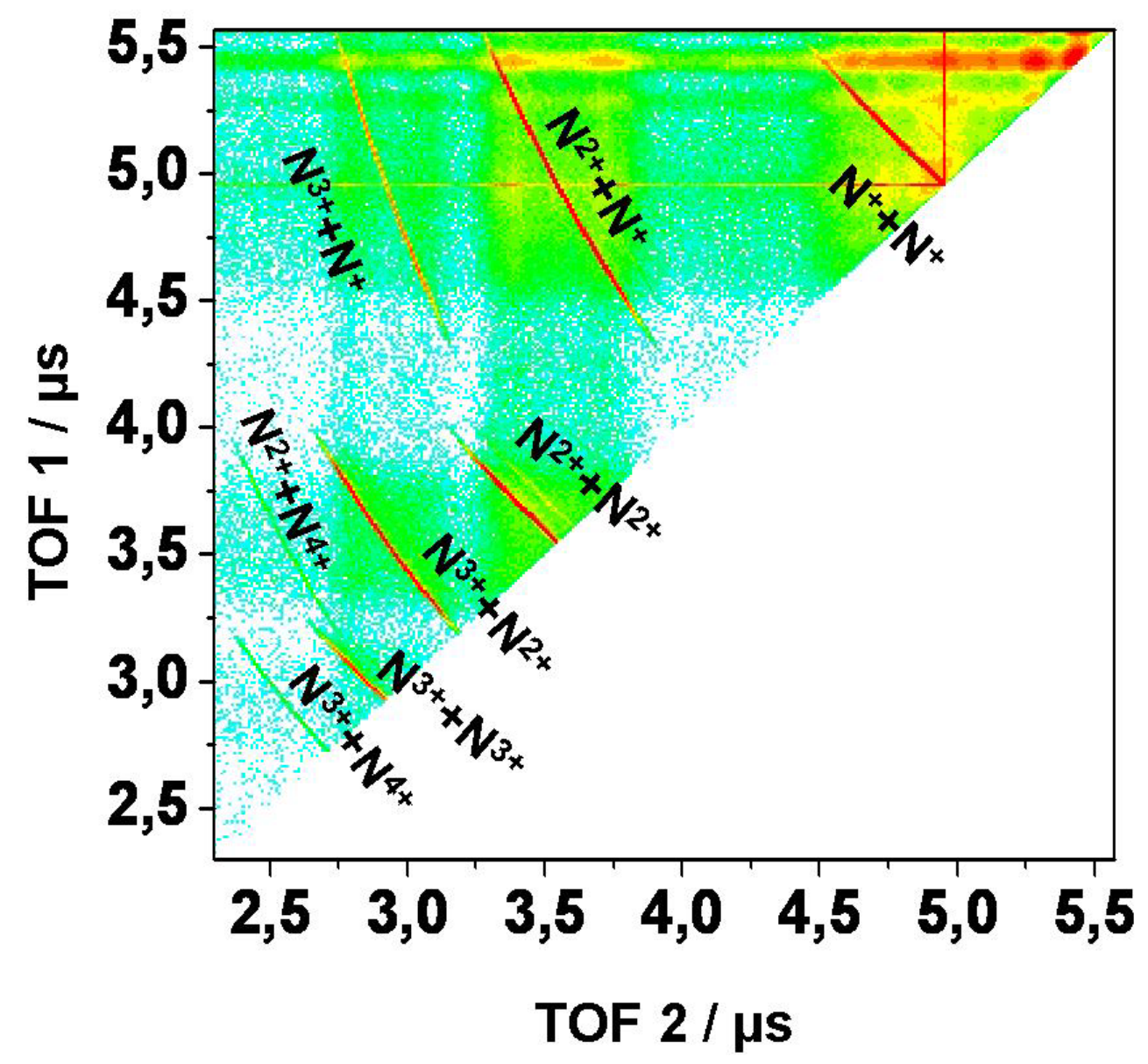

Figure 13 


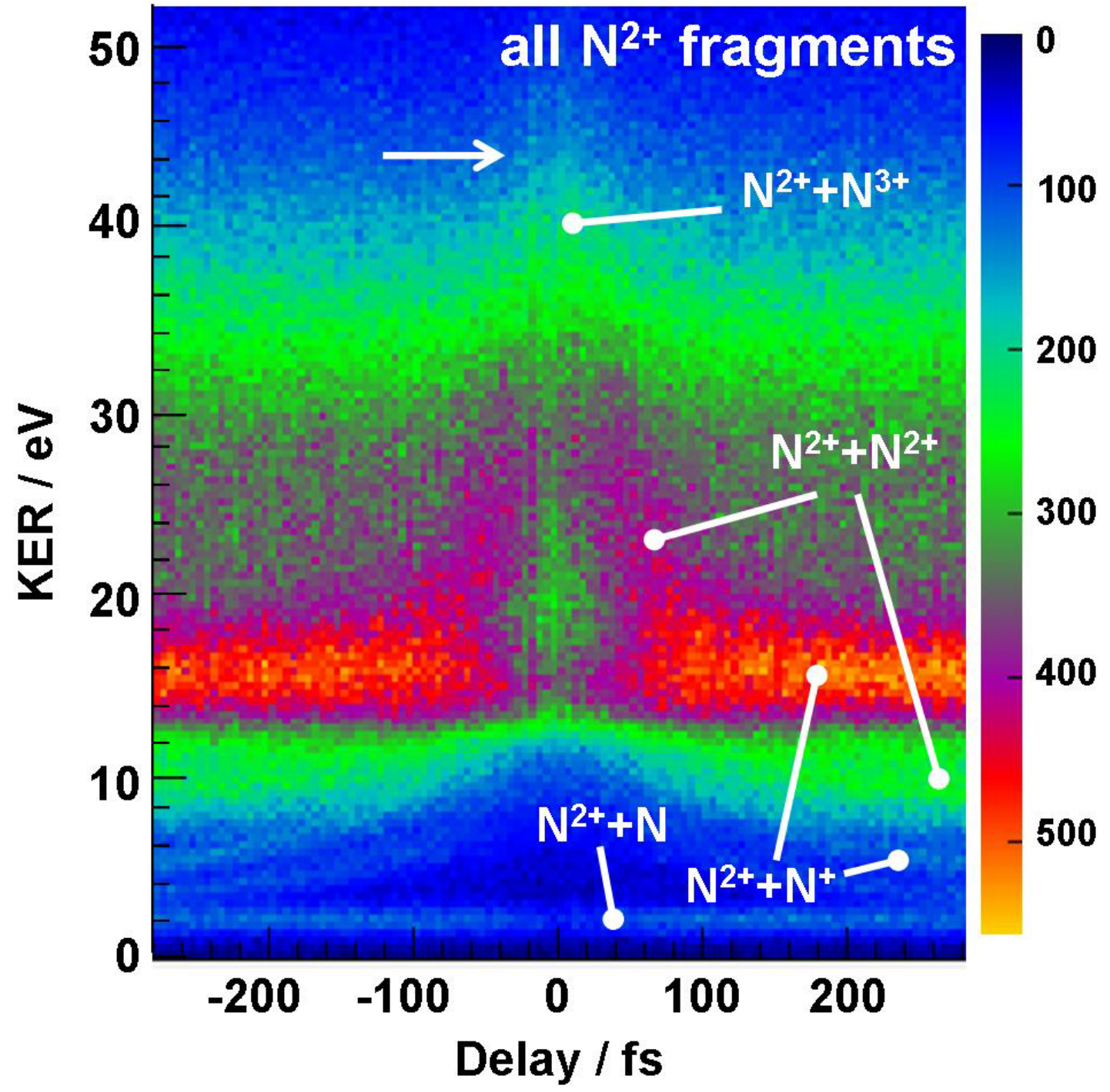

Figure 14 


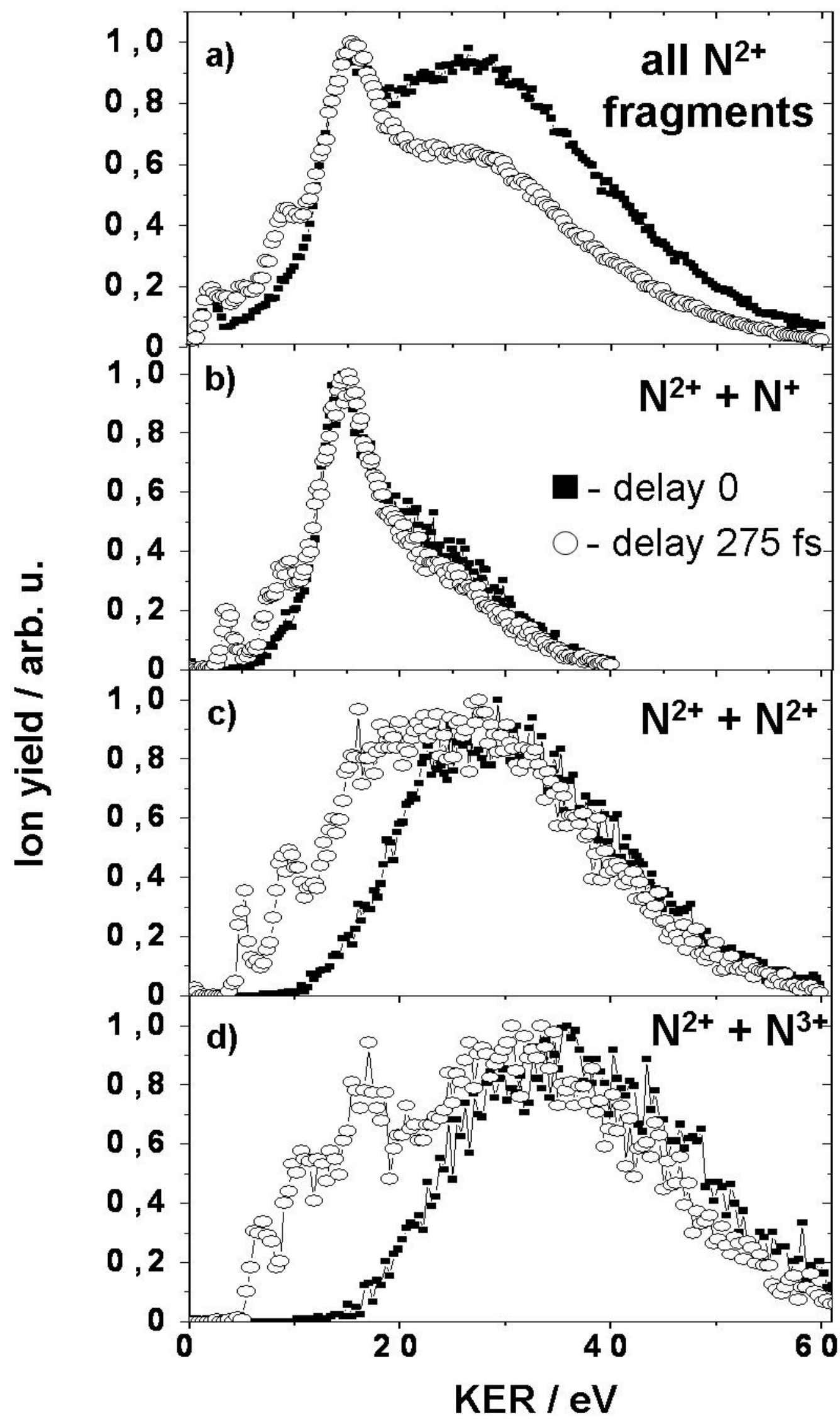

Figure 15 